\title{
Microstructure of natural graphite flakes revealed by oxidation: limitations of XRD and Raman techniques for crystallinity estimates
}

\author{
Heinrich Badenhorst \\ SARChI Chair in Carbon Materials and Technology, Department of Chemical Engineering, University \\ of Pretoria, Lynnwood Road, Pretoria, 0002, South Africa \\ Tel: +27 12420 4173. Fax: +27 12420 2516. E-mail: carbon@up.ac.za
}

\begin{abstract}
The estimation of the active surface area (ASA) of various macrocrystalline graphitic materials is industrially valuable but the microstructures of these materials are still contestable. This in turn has led to difficulties in the unambiguous interpretation of crystallographic measurements with powder X-ray diffraction (pXRD) and Raman spectroscopy as well as their relationship to the ASA. To resolve this issue a systematic approach is required. As a starting point two widely accepted pXRD and Raman methodologies were utilized. Purified, oxidized, natural graphite flakes were extensively examined to elucidate the essential microstructural features. Based on this an illustrative model was formulated as grounds for interpreting the measured crystallite domain sizes. Only one of the crystallographic parameters could be linked to the observed microstructure. For macrocrystalline graphite both techniques are subject to instrumental limitations and should not be used. Due to the non-linearity of the correlations they are prone to measurement uncertainty and should not be used above acceptable limits. In addition, the current inability to distinguish between different defect types leads to ambiguous results. Despite being a single, interrelated
\end{abstract}


crystal the composite nature of the flakes will make it difficult to relate even an ideal, accurate domain size measurement to the ASA.

Keywords: Microstructure, Natural graphite, Defects, X-ray diffraction, Raman spectroscopy, Oxidation, Active surface area

\section{Introduction}

Graphite in its various forms is a very important industrial material, it is utilized in a wide variety of specialized applications. These include high temperature uses where the oxidative reactivity of graphite is very important, such as electric arc furnaces and nuclear reactors. Graphite intercalation compounds are utilized in lithium batteries or as fire retardant additives. These may also be exfoliated and pressed into foils for a variety of uses including fluid seals and heat management.

For these highly graphitic materials the layered structure of the ideal graphite crystal is well established [1]. However, in order to compare materials for a specific application the number of exposed, reactive edge sites are of great importance. This active surface area (ASA) is critical for quantifying properties like oxidative reactivity and intercalation capacity. Due to the nature of these sites and the very low values of the ASA for macrocrystalline graphite, it is difficult to directly measure this parameter accurately and easily.

Graphite and related carbon materials have been the subject of scientific investigation for longer than a century. Despite this fact there is still a fundamental issue that remains, namely the supra-molecular constitution of the various carbon materials $[2,3]$. In particular it is unclear how individual crystallites of varying sizes 
are arranged and interlinked to form the complex microstructures and defects found in different bulk graphite materials.

Traditionally, techniques such as powder X-ray diffraction (pXRD) and Raman spectroscopy have been used to investigate the crystalline structure of these materials. Using pXRD the size of coherently diffracting domains [4] in both the $a-b$ and $c$ directions may be determined. These are thought to be representative of the average crystallite width, $L_{A}$, and average crystallite height, $L_{C}$, of perfectly crystalline regions within the material, as illustrated in Fig. 1A. Using Raman spectroscopy on the other hand the distance between crystalline defects in graphite may be determined [5]. Crystallite edges may also be considered as defects. Thus if the material is assumed to comprise predominantly small crystallites, the inter-defect distance is correlated to the crystallite width, $L_{A}$. Standard methods and correlations have been developed for these techniques $[6,7]$.

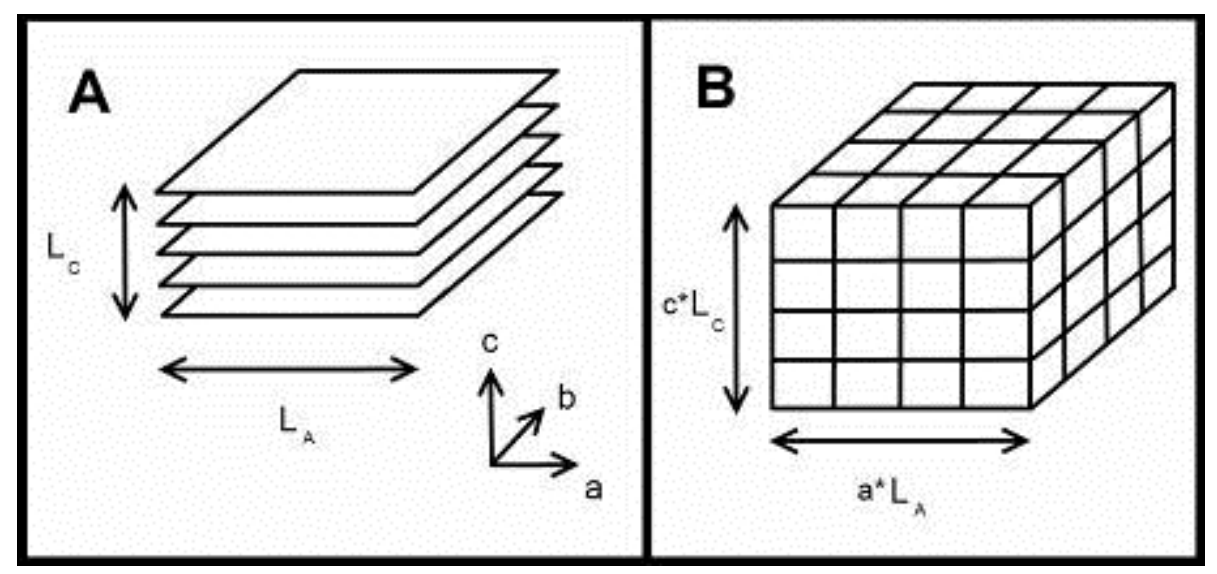

Fig. 1: (A) Basic crystal structural unit, (B) Simplistic microstructural model.

Despite this work the exact definitions of the measured domain sizes and the meanings of their absolute numerical values remain ambiguous $[5,6]$. This dilemma is in part due to the uncertainty regarding the complex microstructure of carbon materials which inhibits firm conclusions regarding the measured values. In addition, a wide variety of correlations and methodologies have been proposed for carbon 
materials. The validity of these approaches for different graphitic materials has not been indisputably established.

It is evident that the ASA will depend heavily on the manner in which these crystalline regions are arranged and interconnected. But if the measured domain sizes could be considered equivalent to the dimensions of discrete crystallites, they could be used to estimate the ASA. Thus in the absence of suitable alternatives, in practice these parameters are either used directly for materials comparison and selection [814], or as input for simplistic microstructural models [15-17]. An example of such a model, shown schematically in Fig. 1B, where $a$ and $c$ integer multiples of the unit cell, was developed to calculate the fraction of edge sites [18]. In this model, atoms at the crystallite boundaries are assumed to be exposed edges. Thus, by using the particle dimensions, crystallographic parameters and surface area measurements, the ASA or edge area may be calculated. This model has been applied to lithium intercalation [18], as well as the oxidation of natural [19] and synthetic [20] graphite. Yet it has not been adequately validated and clearly represents a significant oversimplification.

Crystallographic measurements have been made in numerous other studies on natural graphite [21-30] for a wide variety of applications. Despite being conducted on large, highly crystalline natural graphite flakes, none of these studies reports crystallite domain sizes in either the $a$-b or $c$ directions larger than $1 \mu \mathrm{m}$. Hence a large discrepancy exists between the measured domain sizes and the expected crystallite sizes. Evidently the need exists for a more rigorous understanding of the material microstructure, as well as the unambiguous verification of the limitations of the domain size estimates. 
If this is to be done a systematic approach must be adopted. First it is necessary to select a preferred methodology for parameter determination. For this reason the standardized pXRD methodology proposed by Iwashita et al. [6] was chosen. Unfortunately a standardized methodology for Raman analysis on carbon materials has not yet been proposed, nevertheless the widely used correlation of Cançado et al. [7] was selected as a starting point.

Next these methods were applied to the simplest and most ideal material that is cheaply and readily available. This is done in the hope that the microstructure of this material can be clearly and independently established. Due to the high cost of Kish graphite and highly ordered pyrolytic graphite (HOPG), natural graphite flakes were chosen for this initial investigation. Despite the high purity of the sample, traces of catalytic activity were still found. These were removed via a high temperature heat treatment, that has the added benefit of relieving some of the stress in the material due to processing. Furthermore, due to the beneficiation process and the geological origin of the material, the flakes exhibit regions of structural imperfection. Since oxygen only gasifies graphite at exposed edges or defects, these regions may be largely removed by oxidation, leaving behind only the underlying core flake structure. In addition, this oxidative treatment will reveal crystalline defects such as screw dislocations.

The aim of this investigation was to assess the meaning of the calculated crystallographic parameters and shortcomings of the chosen methodologies, in light of the microstructures of the idealized, primary flake particles. New developments in electron microscopy, such as the use of in-lens detectors, field-emission guns and very low acceleration voltages, allow unparalleled resolution of surface detail $[31,32]$. Using this technique a detailed investigation of the flake microstructure was 
conducted. The key characteristics were identified and related to the crystalline perfection or known defects. This is partially made possible by the consideration of new insights from recent work on defects for nanomaterials [5, 33].

An illustrative model is formulated from these observed characteristics to serve as a basis for the interpretation of the crystallographic parameters. Using this approach it is possible to link one of the crystallographic parameters to the observed microstructural features and defects. The remaining discrepancies are accounted for by considering the limitations of the chosen techniques and correlations. Finally, the relevance of the crystallographic parameters for the determination of the ASA is discussed in light of the proposed model.

\section{Experimental}

The natural graphite (RFL grade, designated PRFL post purification) was obtained from a commercial source: Graphit Kropfmühl (AG Germany). This is a large flake, natural graphite powder. It was purified by the supplier with an acid treatment and a high-temperature soda ash burn up to a reported purity of $>99.9$ mass $\%$. The sample was sieved and only the size fraction between 200 and $250 \mu \mathrm{m}$ was retained. Despite the high purity, the sample still exhibited some residual catalyst activity which was fully removed by a high-temperature heat treatment to $2750{ }^{\circ} \mathrm{C}$ in a furnace under instrument grade Helium, followed by a very slow cool down $\left(<1{ }^{\circ} \mathrm{C} \cdot \mathrm{min}^{-1}\right)$.

All thermal oxidation was conducted in a TA Instruments SDT Q600 thermogravimetric analyzer (TGA) under instrument grade oxygen flowing at 500 ml.min ${ }^{-1}$. The sample was oxidized to a burn-off of around $25 \%$ at $750{ }^{\circ} \mathrm{C}$, at which point the oxidizing atmosphere was rapidly changed to inert. The burn-off level was 
chosen as a compromise between removal of imperfections and degradation of the flake structure. All characterization was done on oxidized flakes.

The impurity constituents of the sample were analysed using an ARL9400 XP+ Sequential X-ray fluorescence (XRF) analyser and Uniquant software. The analysis was done for all elements between $\mathrm{Na}$ and $\mathrm{U}$, but only elements found above the detection limits are reported. The carbon content was calculated by difference.

The powder X-ray diffraction (pXRD) spectrum of the graphite was obtained with 20 mass \% silicon as internal standard. A PANalytical X-pert Pro powder diffractometer with variable divergence and receiving slits coupled to an X'celerator detector using iron-filtered cobalt $\mathrm{K} \alpha$ radiation was used. The use of cobalt $\mathrm{K} \alpha$ radiation was due to the lack of availability of a copper source. The Raman spectra were acquired using a Dilor XY Raman spectrometer using the $\lambda=514.5 \mathrm{~nm}$ laser line of a coherent Innova 90 Ar+-laser. The samples were placed on a glass substrate, the laser intensity was $10 \mathrm{~mW}$ at the sample and no filters were used.

Scanning electron microscope (SEM) images were obtained using an ultra-highresolution field-emission microscope (Zeiss Ultra Plus 55 FEGSEM) equipped with an in-lens detection system and operated at an acceleration voltage of $1 \mathrm{kV}$. A working distance of between 2 and $3 \mathrm{~mm}$ was used. The powder was lightly deposited on carbon tape and examined without any additional sample preparation.

\section{Results and discussion}

\subsection{Impurity analysis}

The sample was analyzed for any impurities using XRF spectroscopy. The composition obtained by the XRF testing is shown in Table 1. 
Table 1: XRF compositional analysis (mass \%)

\begin{tabular}{|c|c|}
\hline & PRFL \\
\hline $\mathrm{Mg}$ & 0.03 \\
\hline $\mathrm{Na}$ & 0.02 \\
\hline $\mathrm{S}$ & 0.01 \\
\hline Carbon & 99.94 \\
\hline
\end{tabular}

The XRF results confirmed the high purity of the sample. At these very low concentrations, close to the detection limits of the machine, the results are only qualitative. The detected impurities may also be the result of post-oxidation contamination. To prove indisputably the absence of catalytically active impurities during oxidation, the samples were extensively examined for evidence of their presence.

Catalytically active particles in graphite can be broadly classified into two specific behaviours [34]: channelling catalysts can be easily identified by their trails, and the catalyst particles responsible for pitting can usually be identified at the bottom of the pit. No traces of catalytic activity were found in the PRFL sample. Impurities that are not catalytically active tend to accumulate at the edges of the particle, especially towards high conversion. Examination of the flakes at high burn-off confirmed the absence of such particles. Thus all surface or microstructural effects are a consequence of the material structure and are not due to catalytic impurities. 


\subsection{Crystallographic domain size measurements}

The use of iron-filtered cobalt $\mathrm{K} \alpha$ radiation is not expected to change the shape of the pXRD spectra obtained. However, the use of the higher wavelength from $\mathrm{CoK} \alpha$ radiation $(0.17903 \mathrm{~nm})$ as opposed to the value for copper $(0.15418 \mathrm{~nm})$ will result in a shift of the peak positions to higher angles. As will be demonstrated, the material exhibits a full width at half maximum intensity (FWHM) for all reflections of less than $0.5^{\circ}$. In this case the standard method [6] does not require the profiles to be corrected for Lorentz, polarization, absorption or carbon-scattering factors. Furthermore, as will be seen, all but the (002) peak are easily resolved into the $\mathrm{K} \alpha_{1}$ and $\mathrm{K} \alpha_{2}$ doublets, and therefore separation correction is applied only to the (002) peak. The regions of the pXRD spectra for the three most relevant peaks, namely (002), (004) and (110), are shown in Fig. 2A through $\mathrm{C}$ respectively for the PRFL natural graphite sample.

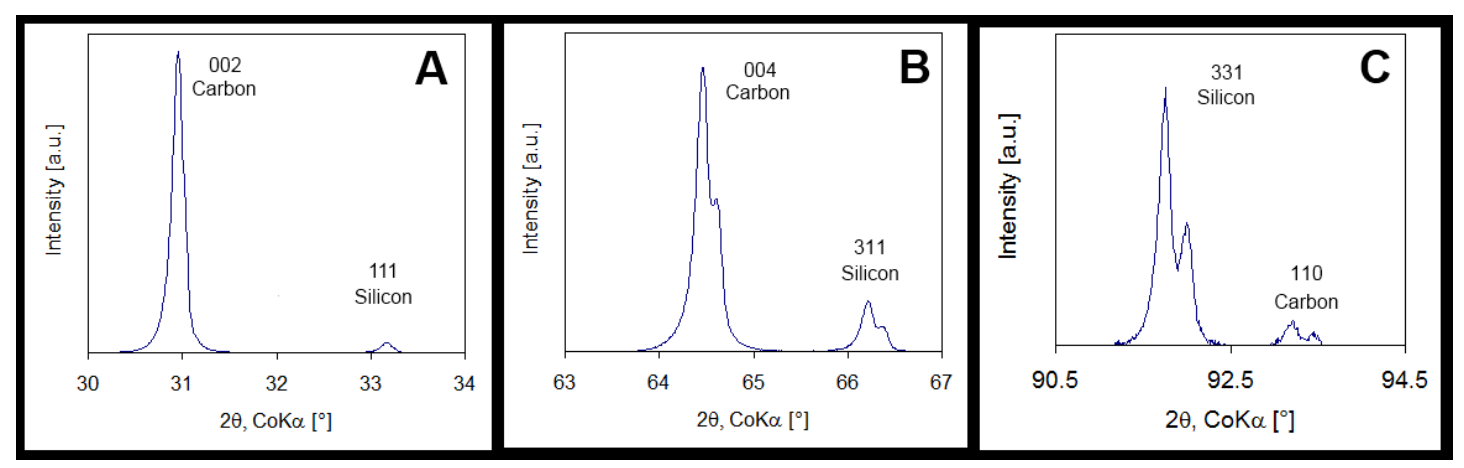

Fig. 2: Raw pXRD spectra for PRFL graphite.

The near-perfect crystallinity of this graphite may be confirmed by the interlayer spacing which is calculated to be $\mathrm{d}_{002}=0.3354 \mathrm{~nm}$, in exact accordance with the theoretically expected value. However, two significant departures from the example spectra given in the standard method [6] are visible. Firstly, the intensity of the (002) peak is remarkably higher than the silicon reference. This effect is also present for the 
(004) peak, although not as pronounced. Secondly, the (110) reflection is significantly lower than expected.

The primary cause of these two deviations is the preferred orientation of the graphite flakes. Due to their large size, the flakes tend to lie flat, giving rise to a significant bias in terms of the basal plane reflections. The converse is also true: the preferred orientation leads to a reduction in the intensities for reflections perpendicular to the basal plane, such as the (110) direction. Conventionally, this issue is resolved by grinding the sample into a fine powder [4, 35]. However, this is not a preferred option for highly crystalline graphite since any milling will inevitably degrade the crystal structure $[15,36,37]$.

Nonetheless, in accordance with the standardized method and to establish some degree of homogeneity, the sample was lightly mixed with the silicon standard in an agate mortar. SEM examination of this mixture indicated very little damage to the bulk flake bodies. Despite these complications, it is still possible to calculate the crystallographic parameters from the observed pXRD spectrum. Each peak was fitted using the standard Lorentzian technique. For easier visualization the fitted peaks were normalized and these are shown in Fig. 3.

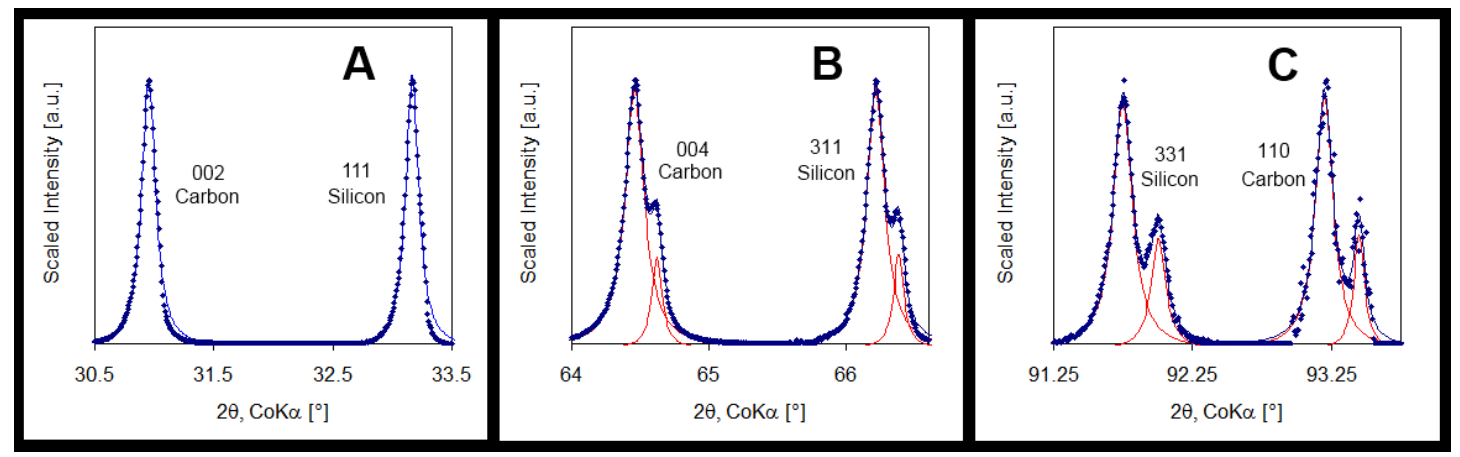

Fig. 3: Normalized pXRD spectra for PRFL graphite.

The fits are only illustrative and FWHM measurements were done manually. However, in this case expressions given by the standard method for the crystallite size 
cannot be used since they already contain the appropriate parameters for CuK $\alpha$ radiation. Nonetheless, as stated, the formulas were calculated using the simple Scherrer equation with the shape factor $K$ set to one. Thus crystallite sizes can be calculated directly from the Scherrer equation using the correct values for $\mathrm{CoK} \alpha$ radiation. The FWHM must still be corrected for instrument broadening using the silicon standard as stipulated. The results are shown in Table 2.

Table 2: Observed FWHM and pXRD domain sizes for PRFL graphite

\begin{tabular}{|c|c|c|c|}
\hline Peak & Silicon & Graphite & Domain size \\
& FWHM $\left(^{\circ}\right)$ & FWHM $\left(^{\circ}\right)$ & $(\mathbf{n m})$ \\
\hline$(002):$ Crystallite domain height & 0.1443 & 0.1514 & $L_{C}=595$ \\
\hline$(004):$ Crystallite domain height & 0.1639 & 0.1865 & $L_{C}=385$ \\
\hline$(110):$ Crystallite domain width & 0.1652 & 0.1760 & $L_{A}=793$ \\
\hline
\end{tabular}

The two significant peak regions of the measured Raman spectra for PRFL graphite are given in Fig. 4A and B respectively.

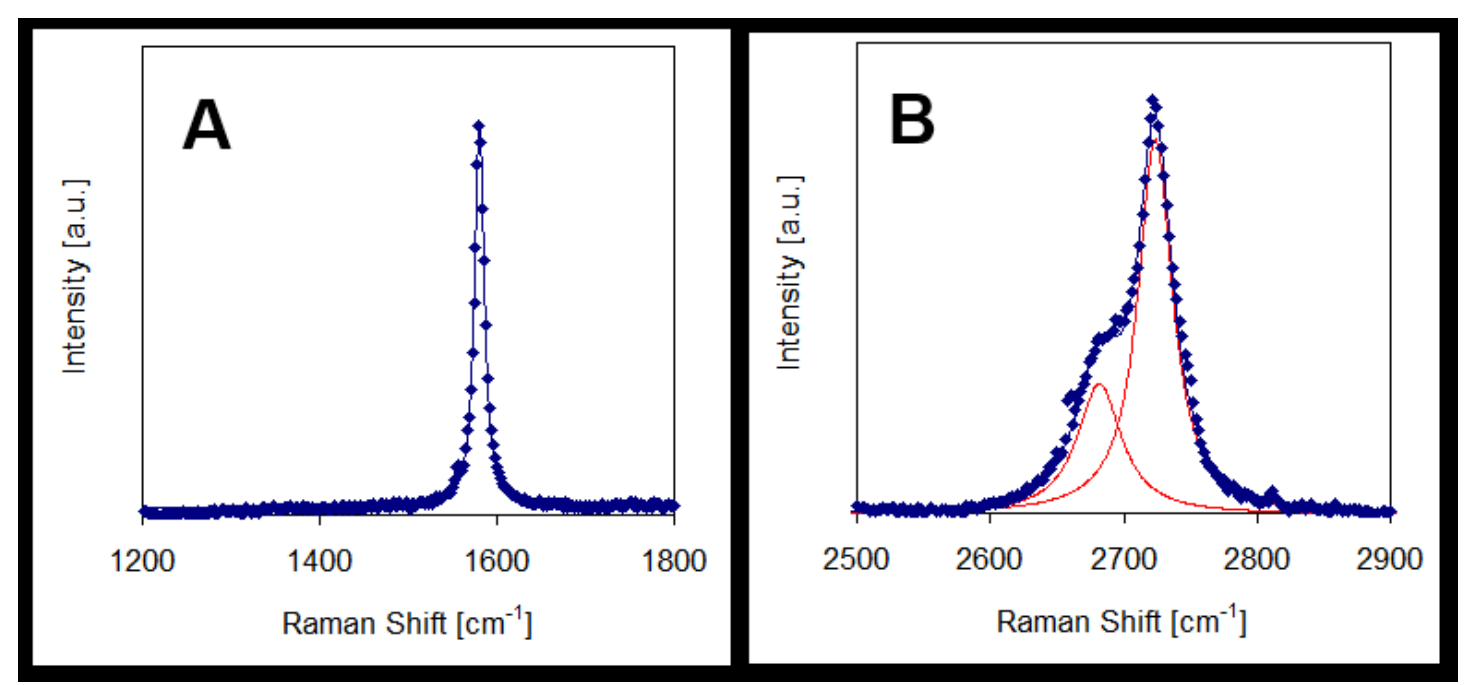

Fig. 4: (A) D and G (B) 2D or G' Raman peaks for PRFL graphite.

The observed spectra are in accordance with those expected for highly crystalline graphite [5]. The FWHM of the G peak $\left(1580 \mathrm{~cm}^{-1}\right)$ is narrow, the $\mathrm{D}^{\prime}$ peak $\left(1620 \mathrm{~cm}^{-}\right.$ 
$\left.{ }^{1}\right)$ is absent, the doublet structure of the $2 \mathrm{D}\left(\right.$ or $\left.\mathrm{G}^{\prime}, \sim 2700 \mathrm{~cm}^{-1}\right)$ peak is clear and the $\mathrm{D}$ $\left(1360 \mathrm{~cm}^{-1}\right)$ peak appears absent. Closer examination of the D peak region does reveal that a very small peak is present in the noise, as can be seen in Fig. 5.

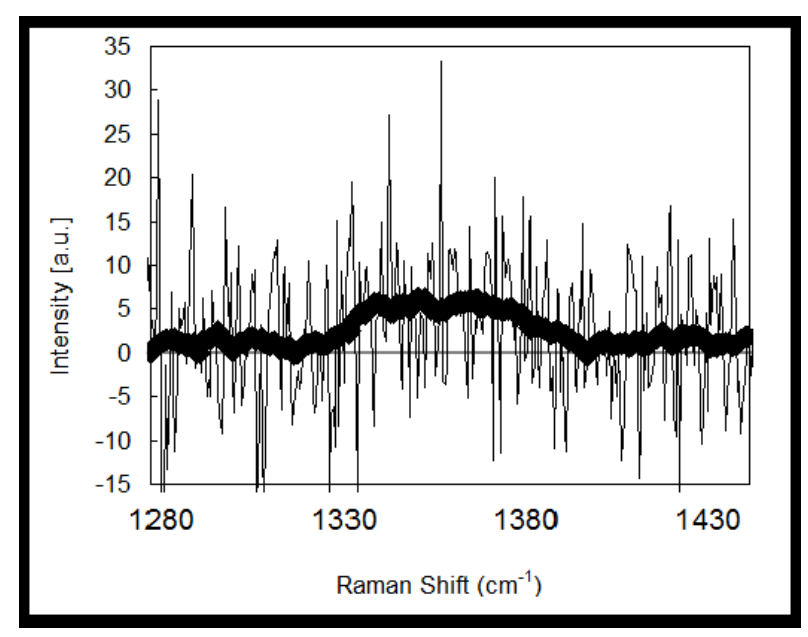

Fig. 5: D peak of Raman spectra for PRFL graphite.

The integrated intensity of the heavily filtered D peak (thick black line in Fig. 5) and the $\mathrm{G}$ peak can be used in the adjusted correlation [7] of Tuinstra and Koenig [38] to calculate the crystallite domain width. This calculation yields a value of $\mathrm{L}_{\mathrm{A}}=998$ $\mathrm{nm}$. This measurement was repeated five times on the pinacoid faces of randomly selected particles and identical spectra were found.

\subsection{Microstructural investigation}

If the unoxidized, purified flakes are examined, the complex dodecagonal and coalesced dodecagonal surface structures characteristic of graphite crystals $[39,40]$ are evident, as in Fig. 6A. 


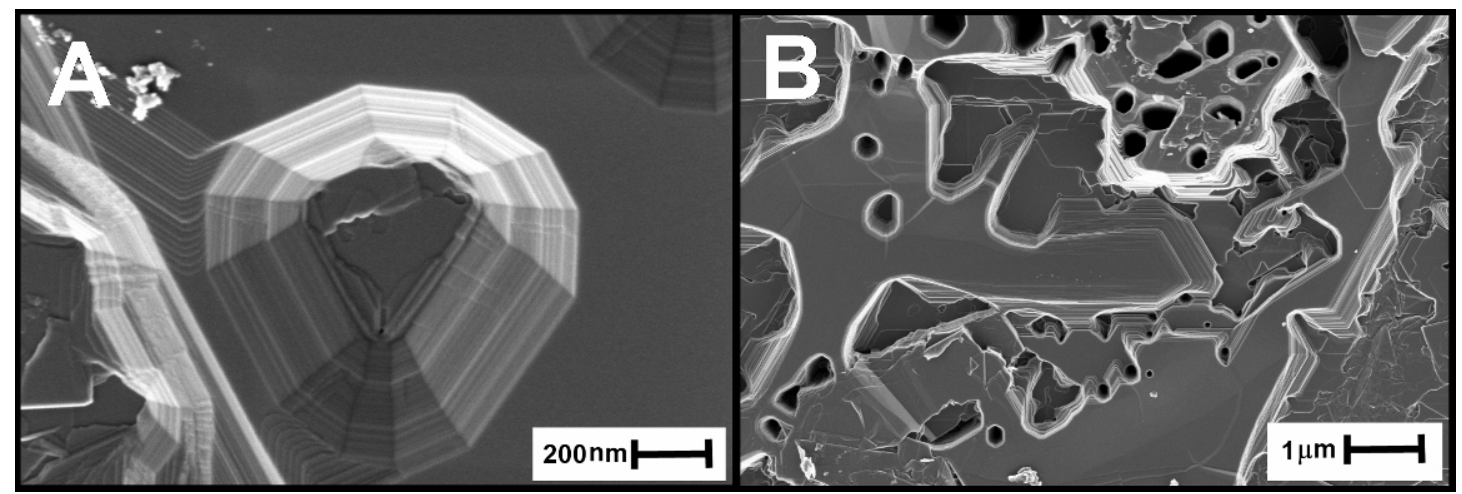

Fig. 6: (A) Dodecagonal and (B) coalesced dodecagonal, atypical surface structures in unoxidized, heat treated material.

More complex, atypical surface structures are also found, such as those visible in Fig. 6B. These structures were not discernable in the material before heat treatment. Since SEM can only discern topographic differences, it implies that these regions were filled. This gives an indication that the flake graphite was formed with inclusions trapped in the larger structure, which is not unexpected given its geological origin. These diminutive structural imperfections have a high ASA and defect density, whilst being mainly sporadically located on the outer surfaces of the flakes. They are largely removed during the oxidation step, leaving only the core flake body as shown in Fig. 7A. This image and all subsequent SEM images depict oxidized PRFL flakes.

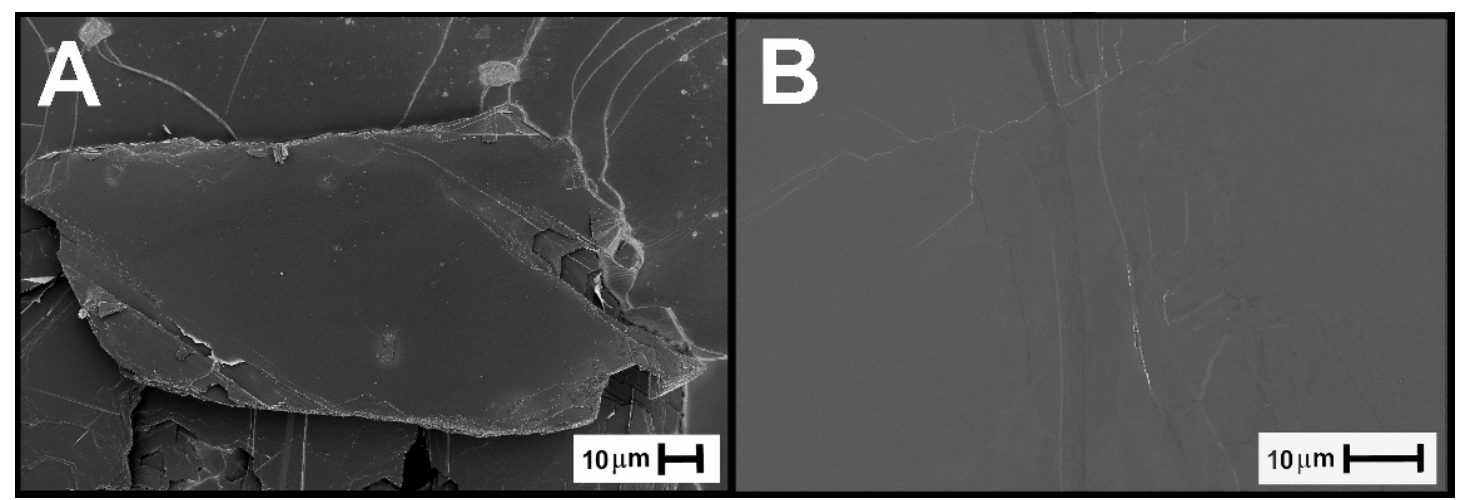

Fig. 7: (A) Oxidized PRFL flake particle and (B) close-up of basal surface.

When the basal surface is examined more closely as in Fig. $7 \mathrm{~B}$, it is found to be fully intact and smooth across very large areas $(\sim 100 \mu \mathrm{m})$. Only some small surface 
steps and ridges are noticeable in this image. When the edge structures are inspected, the characteristic $120^{\circ}$ angles expected for pristine graphite crystals are readily identifiable as in Fig. 8A.

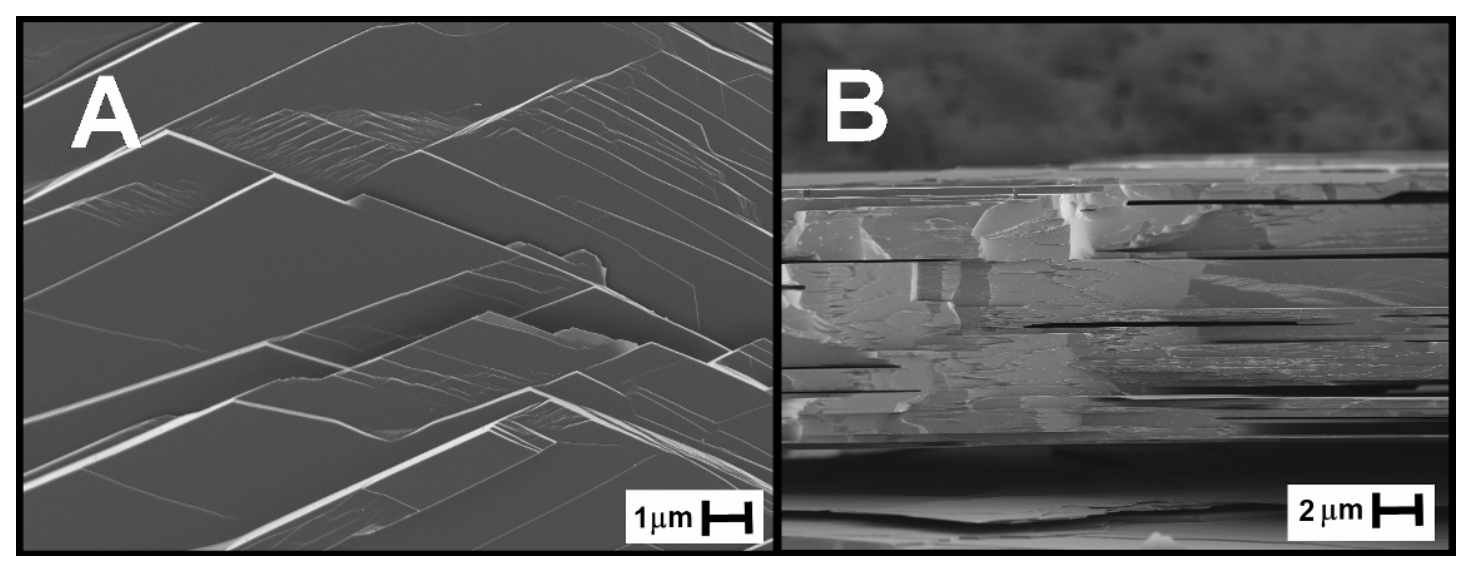

Fig. 8: (A) Oxidized PRFL flake edges from above and (B) from edge-on.

Furthermore, when the edges are examined face-on, they are found to be smooth and fairly flat across distances of several micrometers, shown in Fig. 8B. In some instances natural graphite flakes are considered to be a single crystals [26, 41-43]. Based on the smooth, flat basal surface across the entire flake width and the large, regular edge faces, it appears that this may very well be the case.

However, domain size measurements determined by pXRD and Raman are significantly smaller than these observations. In the $a-b$ direction the estimates are lower than one micrometer whereas the regions of intact basal surface are two orders of magnitude larger. Whilst in the $c$ direction the estimates are around $500 \mathrm{~nm}$ but the flake thickness is on the order of several micrometers. An extensive survey of the material does reveal that a few easily identifiable defect structures are present, such as the screw dislocation shown in Fig. 9A. 


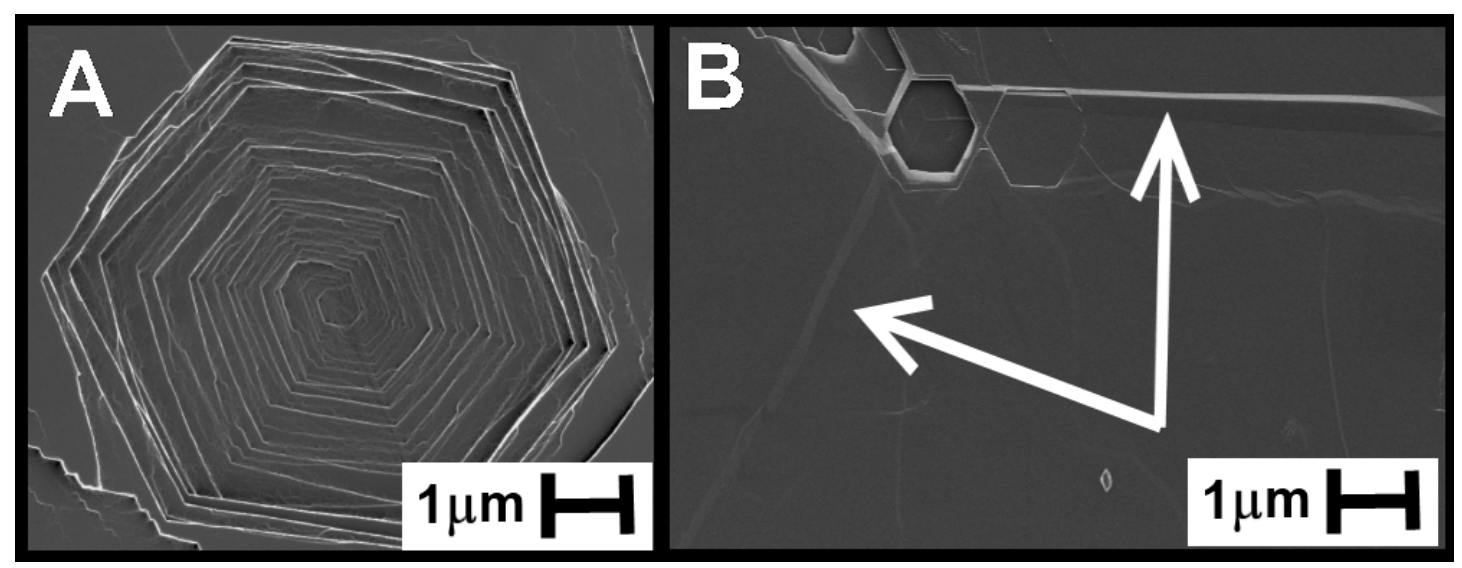

Fig. 9: (A) Screw dislocation and (B) twinning bands in oxidized PRFL graphite.

These dislocations are easily distinguishable by the large pits that form during oxidation with a characteristic corkscrew shape [44]. In general, no more than a few discrete occurrences of these defects were found in any given flake. A more prevalent defect is twinning, which is derived by a rotation along the armchair direction of the graphite crystal. These defects usually occur in pairs, forming the characteristic twinning bands visible in Fig. 9B.

The fixed orientation of the twinning band allows identification of the crystal orientation in surrounding edges [45]. Thus it is clear that all the edges or surface steps visible in Fig. 9B are oriented along the armchair edge. It was found that the majority of edges had this orientation, which is consistent with the findings of Thomas [45]. Due to the reaction anisotropy of the two edge structures, Thomas found that during oxidation below $900{ }^{\circ} \mathrm{C}$, oxidation along the armchair edge dominates.

These defects do not necessarily negate the assertion that the natural graphite is a single crystal. However, other phenomena are harder to explain from this point of view. For example, extensive arrays of steps are found across edge faces, as shown in Fig. 10A. 


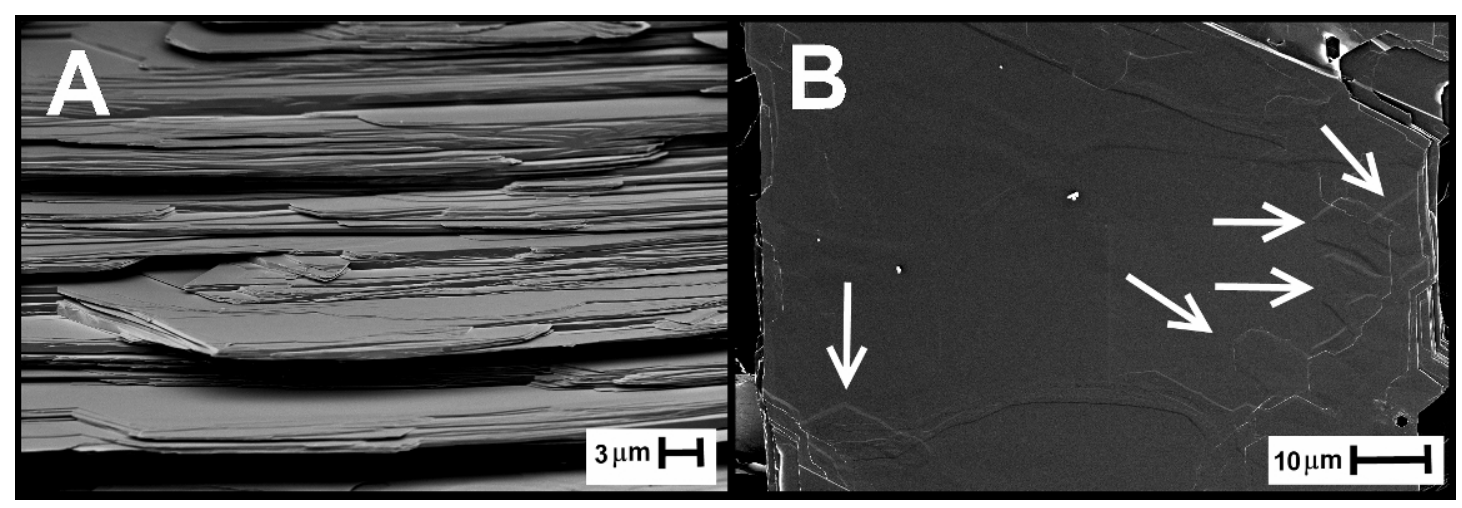

Fig. 10: (A) Edge steps and (B) polygonal surface ridges.

If the entire particle constitutes a single crystal, one would expect homogenous oxidation across the entire thickness of the flake, but this is never the case. Nonetheless, consideration of Fig. 8A indicates that multiple overlying layers are present at this edge too, with a terraced structure similar to that visible in Fig. 10A. Yet all the edges are aligned, thus the underlying crystal structures must be too.

In addition, surface ridges are evident on the basal surfaces of some flakes, as indicated in Fig. 10B. Twinning is generally induced by deformation. The polygonal nature of these ridges makes it unlikely that they were formed via this mechanism. Thus the nature and cause of these edges and ridges require additional consideration.

\subsection{The role of defects}

The discrepancy between the observed microstructure and measured domain sizes may be caused by defects which are not necessarily exposed by oxidation. Thus it is essential first to consider briefly all the defects that exist in graphite. Four possible defect structures are generally found in graphite [46, 47], namely:
i) Basal dislocations
ii) Non-basal edge dislocations
iii) Prismatic screw dislocations 
iv) Prismatic edge dislocations

Due to the fact that the breaking of carbon-carbon bonds is required for non-basal dislocations, the existence of type (ii) defects is highly unlikely [46, 48]. Given the very weak van der Waals bonding between adjacent layers, however, basal dislocations are very likely and a multitude have been documented $[1,48]$, especially in deformed specimens. These defects may be divided into two groups [48], namely (1) stacking disorder and (2) rotations between successive sheets.

One of the most energetically favourable stacking faults is the abcabc stacking sequence found in rhombohedral graphite. This defect is commonly found in ground natural graphite that has been extensively deformed [49]. This form of graphite can be easily identified by its (100) and (110) reflections at $2 \theta=50.9^{\circ}$ and $54.4^{\circ}(\mathrm{CoK} \alpha)$ respectively. Recent work [50-52] indicates that it is possible to detect the $a b c$ stacking sequence in three- to four-layered graphene using Raman spectroscopy. However, the variations are very subtle and work on bulk materials has shown that even at high proportions no appreciable differences can be detected in the Raman spectra [53]. Rhombohedral graphite is thermodynamically unstable above temperatures of around $2000{ }^{\circ} \mathrm{C}$ [54]. Given the heat treatment to which this material has been subjected, it is not expected to contain any rhombohedral phase. This is confirmed by the XRD spectrum which lacks the rhombohedral peaks, even post mixing in the agate mortar. The absence of this stacking fault would preclude the presence of any less energetically favourable stacking faults. Hence it may be concluded that there are no group (1) stacking defects in this material.

The second group of type (i) defects denote a rotation of the basal planes relative to each other and the formation of turbostratic graphite. This structure represents a 
gross breakdown in the periodicity of the lattice in all but the $c$-direction. As such, this will lead to a reduction in all but the $(00 l)$ reflections $[55,56]$. The pXRD spectrum does indeed show a comparative decrease in the (110) reflection, but this may be attributed to the preferred orientation of the flakes, as discussed earlier. Nonetheless the measured domain size in this direction remains fairly large, making misalignment of individual layers doubtful. Recent work has shown $[33,57]$ that for turbostratic graphite the 2D Raman peak will tend towards a single peak centered at around $2710 \mathrm{~cm}^{-1}$. This is similar to the peak for single-layer graphene but with a larger line width. As can be seen from Fig. 4B, this is clearly not the case and it may be concluded that the natural graphite has virtually no detectable turbostratic disorder. This conclusion is further supported by the virtual absence of the D peak, which is almost invariably present in turbostratic materials [58-60], as well as the consistent $120^{\circ}$ angles of the edges seen in Fig. 8A. This does however not preclude the existence of much larger twist grain boundaries which may very well be present.

The next possible defect is type (iii), prismatic screw dislocations. As mentioned in the previous section, these defects have been found in the PRFL material but at comparatively low densities. In addition to screw dislocations, oxidative attack is possible at point defects such as a lattice vacancies [61]. However, these vacancies are generally only induced by neutron irradiation or rapid quenching from very high temperatures [62] and as such are unlikely to exist in the sample under consideration.

The final defect is type (iv), prismatic edge dislocations, of which the classical representation is shown schematically in Fig. 11A [48]. 


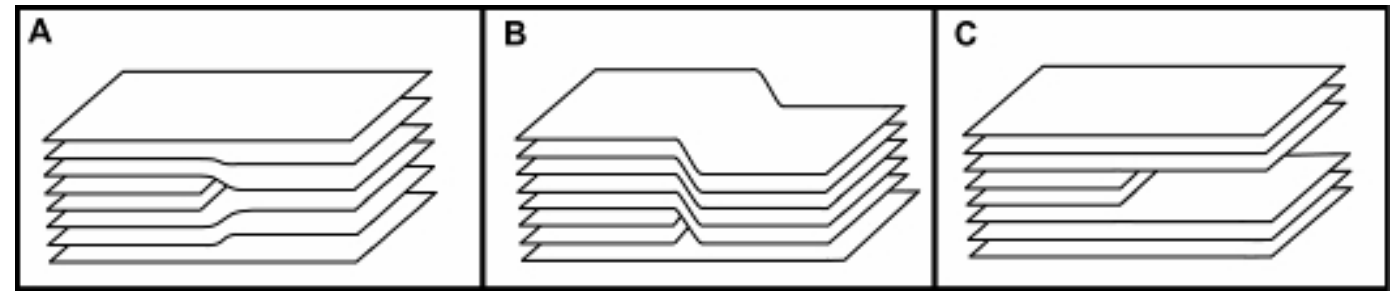

Fig. 11: Prismatic edge dislocation in graphite.

In early studies these structures were formed by the precipitation of interstitials or vacancies and are therefore usually found in rapidly quenched or irradiated graphite $[62,63]$. The point defects will coalesce to form small layers, or cavities, resulting in prismatic dislocation loops. Given the history of the material under consideration, the occurrence of defect loops on these grounds are unlikely. However, flake natural graphite is formed under high pressure and temperature conditions during the creation of metamorphosed siliceous or calcareous sediments [64]. It is not inconceivable that during these extreme processes graphite crystallizes with prismatic edge dislocations trapped within the structure, perhaps due to the inclusion of other minerals. Furthermore, it is not impossible that these structures will have several hundred or perhaps even thousands of layers, as opposed to the two layers schematically represented in Fig. 11.

The height of these defects is an important point to consider. If the dislocations are very small and consist of only a few layers, the defects will be infinitesimal and not directly observable with the SEM. If they are located within the bulk of the material, the situation shown in Fig. 11A may indeed prevail. Evidence for the existence of these defects has been found, but they comprise more than a few layers, as is visible in Fig. 12. 


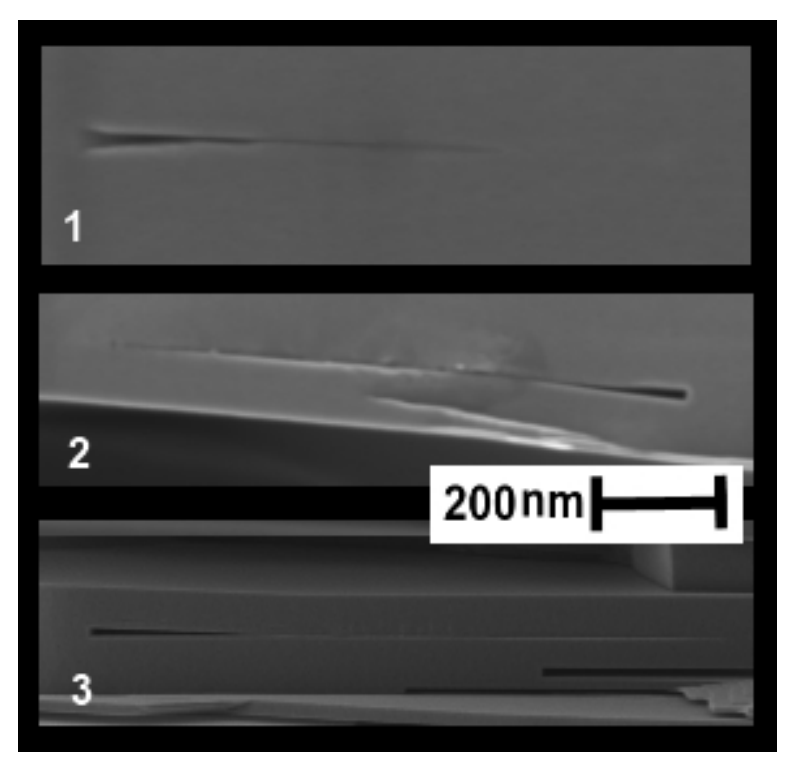

Fig. 12: Thin prismatic edge dislocations in graphite.

A very small slit-like pore is visible which gradually tapers down and is finally no longer visible. In other cases, such as near the top surface, if the number of layers in the defect is sufficiently high, the uneven stacking on the right-hand side of Fig. 11A may not be stable. Instead it is possible that the stack will collapse, leading to the formation of a surface ridge and subsequent shear movement of the planes, as shown in Fig. 11B. The bends produced by these defects are pure tilt boundaries, similar to twins.

It is important to notice that the shear dislocation caused by stack collapse in Fig. 11B does not represent a type (i) stacking order defect. Rather, this is a relative shift in two blocks having continuous ababab stacking, without any rotation. Thus it represents a single disruption in the stacking order. The size of the relative shift is dependent on the height and angle of the fault. However, even in the smallest flaws this misalignment may be enough to disrupt homogeneous reactivity across an edge face, leading to a step along the edge. This accounts in part for the terraced structures found in Figs 8A and 10A. The terrace layers have undergone horizontal slip relative to each other but no rotation, and thus the edges are still parallel along the 
crystallographic directions. As mentioned, these collapsed dislocations will result in a surface ridge, two examples of which are shown in Fig. 13 A and B.

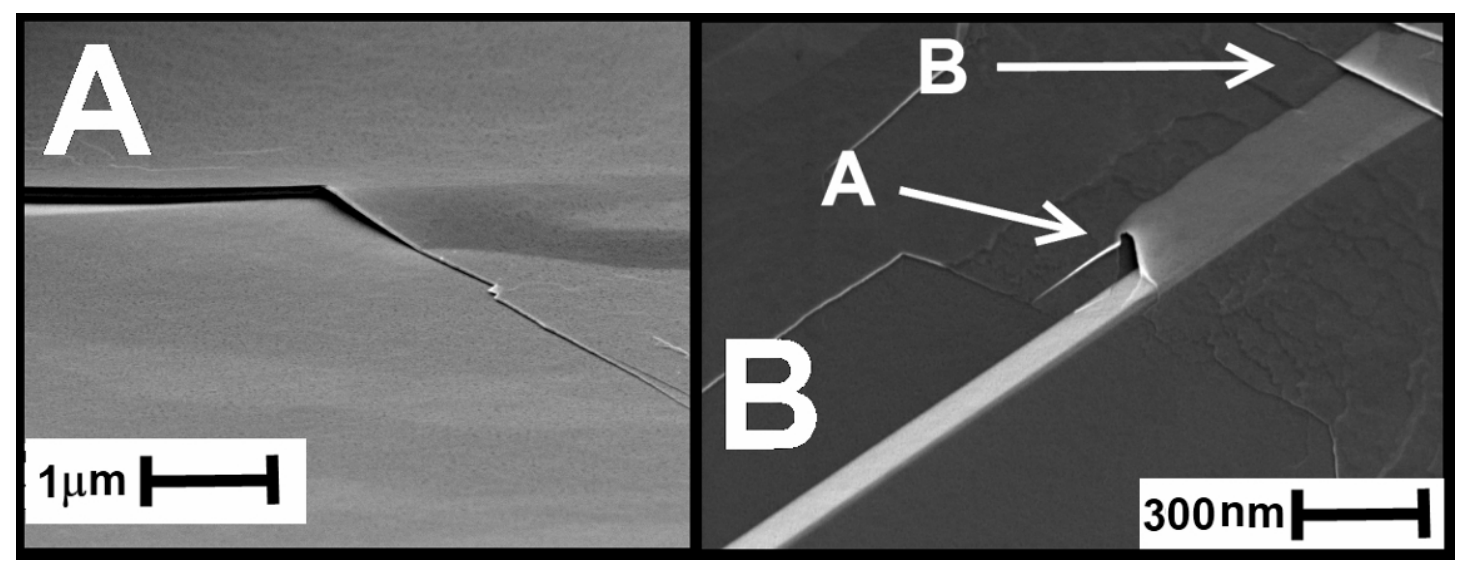

Fig. 13: (A) and (B) Surface ridges due to underlying edge dislocations.

Typically twinning occurs at a single angle of approximately $20^{\circ}$ [45] but variations on this angle, usually less, have been observed [65]. The two ridges visible at points $\mathrm{A}$ and $\mathrm{B}$ in Fig. 13B have different slopes, as indicated by their relative lengths. This implies that each has a different angle, despite being part of the same twin. Other more complex twinning phenomenon are observed, especially when these defects are viewed at the particle edge, as in Fig. 14.

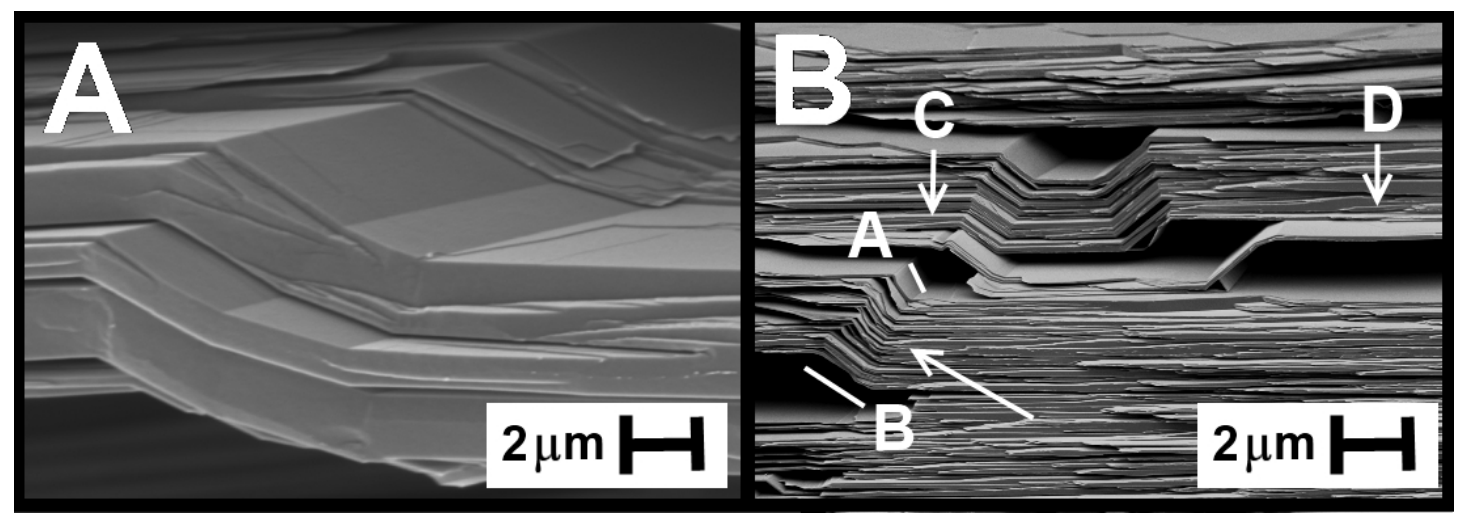

Fig. 14: (A) and (B) Complex twinning behaviour.

In Fig. 14A, two sequential rotations are visible within a single step, whereas in Fig. 14B a gradual rotation of the flaw angle occurs from point $\mathrm{A}$ to point $\mathrm{B}$. In addition, asymmetric banding is visible between points $C$ and D. This may be caused 
by edge dislocations of different sizes on either side of the rotations. A particularly relevant twinning formation is shown in the edge-on view of Fig. 15, where the basal surface extends into the page.

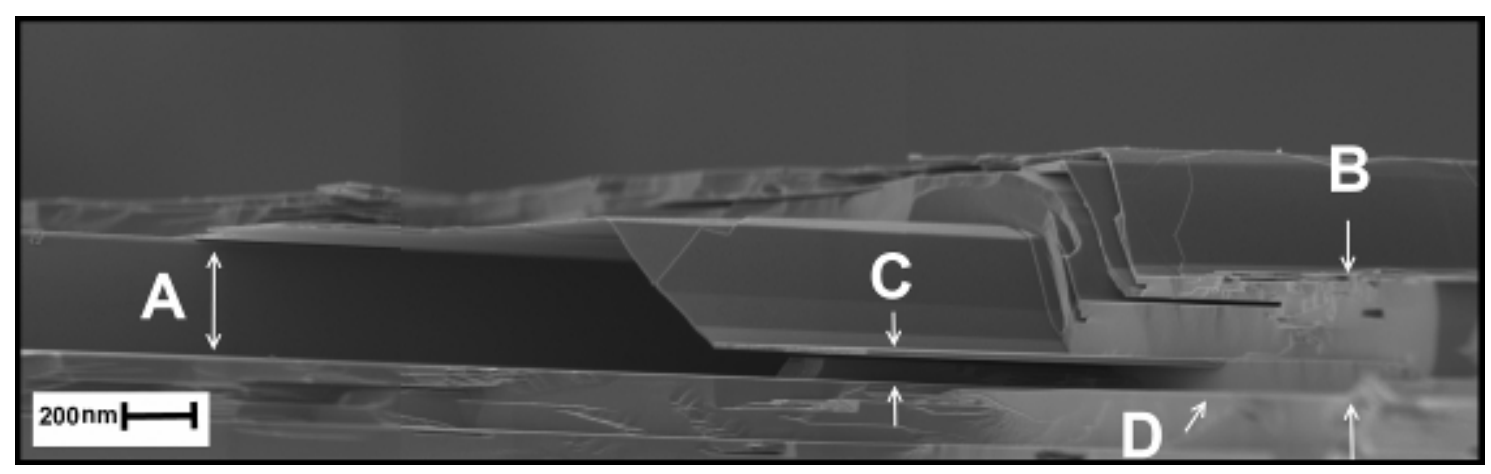

Fig. 15: Complex twinning with underlying edge dislocation.

The entire surface at point A represents an underlying edge dislocation. The top layer undergoes multiple rotations and eventually links up with the extension of the original crystal at point B which has a homogenous edge face. It should be noted that it would be impossible to form such a structure through deformation. These formations explain the existence of the polygonal surface ridges visible in Fig. 10B. The underlying structures causing these ridges can be virtually any shape but must have angles consistent with the graphite lattice. The overhang at point $\mathrm{C}$ of Fig. 15 is an example of the edge dislocation shown in Fig. 11C. In this case the overhang is supported by the edge dislocation at point $\mathrm{D}$. Thus the individual flakes are intricate but interrelated lattices, characterized by complex twinning or folds in the sheets resulting in a relative shift and edge step formation.

\subsection{Microstructural model}

Based on these observations it is possible to formulate a microstructural representation of the natural graphite. The polygonal nature of the flake structure is clearly evident in Fig. 16A. 


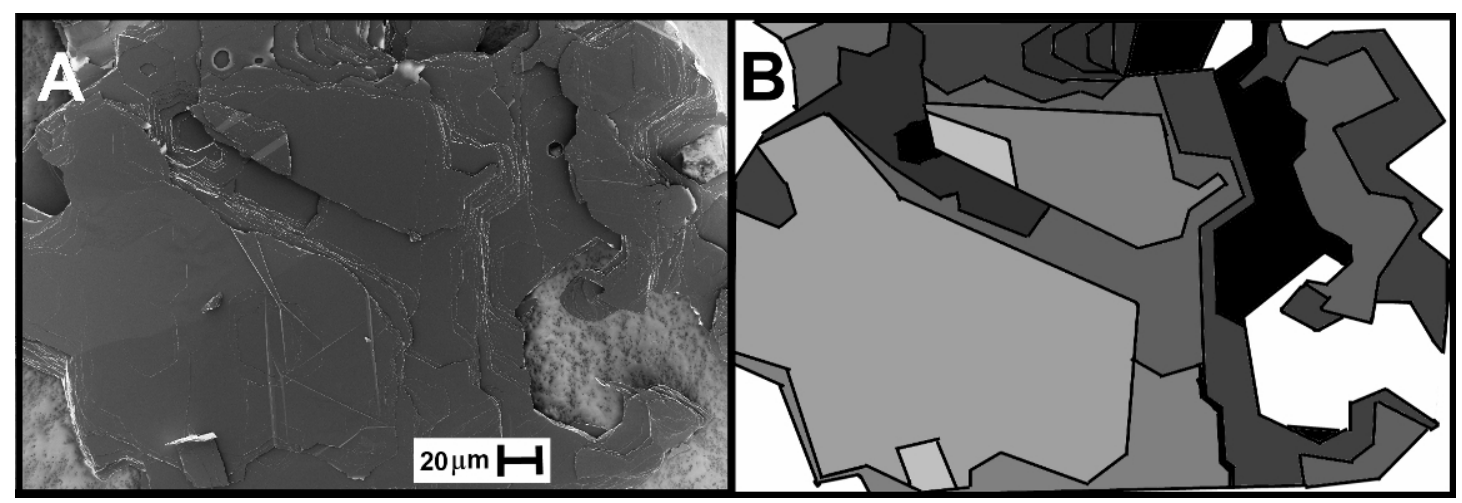

Fig. 16: (A) Polygonal flake structure and (B) schematic representation (lighter shades indicate increasingly elevated segments).

An individual flake consists of collections of fairly regular polygons which comprise multiple, continuously stacked graphitic layers. This is schematically represented in Fig. 16B in which progressively lighter shades indicate increasingly elevated segments. The polygons all contain the characteristic $120^{\circ}, 60^{\circ}$ or $30^{\circ}$ edge angles, but their shapes and arrangements are fairly random. Yet the entire flake is interconnected since it does not disintegrate into separate pieces. This polygonized and interlinked flake structure is clearly visible in other natural graphite crystals [66]. These morphologies can be qualitatively explained by complex crystal growth phenomena. Due to disclinations and elastic instability crystal growth proceeds along a macrospiral which interlinks the entire structure whilst polygonized blocks develop independently. The interwoven layered structure is typically represented by the formation shown in Fig. 17A. 


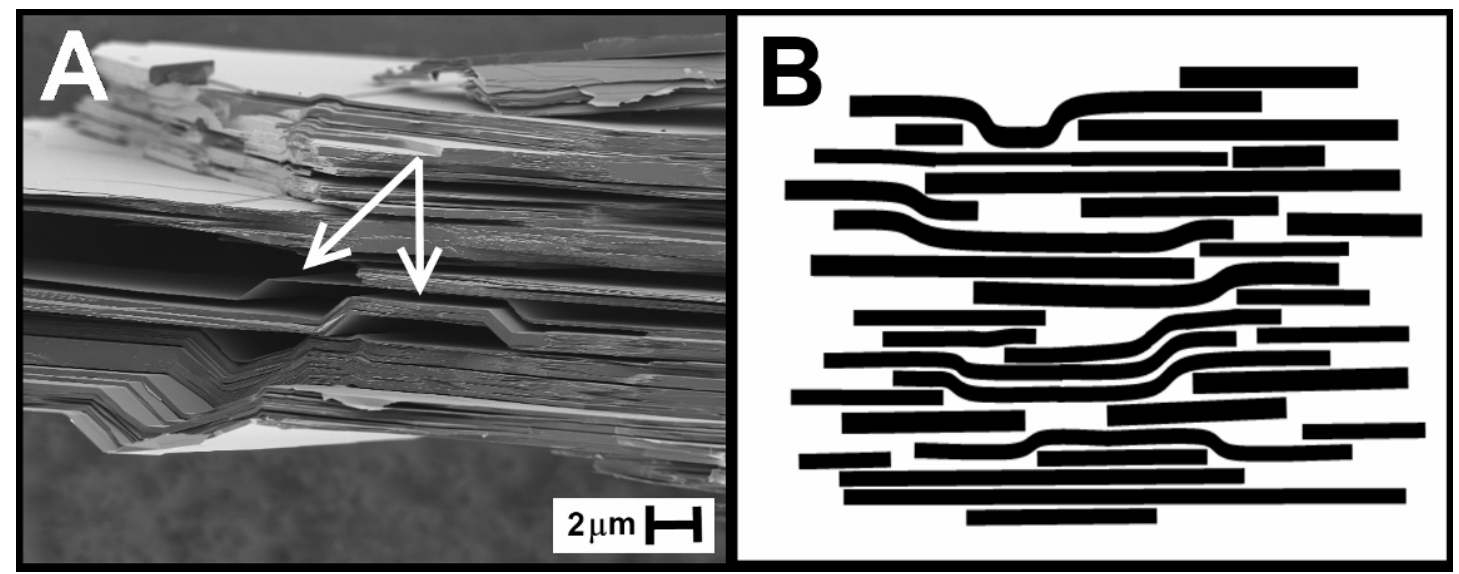

Fig. 17: (A) Representative layered structure and (B) schematic representation of interlinked flake segments with resulting slit-shaped pores.

When viewed edge on, a random stacking arrangement is expected, as shown schematically in Fig. 17B. The slit-like pores are caused by prismatic edge dislocations such as those shown schematically in Figs 11B and C and the associated complex twinning behaviour. These may have been induced by the presence of other minerals which were removed during heat treatment, or could also have been caused by damage during beneficiation. However it should also be noted that the defect structures located within the macro flake body at the white arrows, show no corresponding surface structures above or below and exist independently within the flake. It would be impossible to create such a defect through deformation alone.

Large regions of overarching connectivity exist linking together disparate segments separated by the slit-like pores. This maintains the same crystallographic orientation throughout, albeit with some horizontal shifting relative to each other but with little to no rotation. This configuration accounts for the structures sometimes visible in expanded intercalated graphite, such as those shown in Fig. $18 \mathrm{~A}$ and B. 


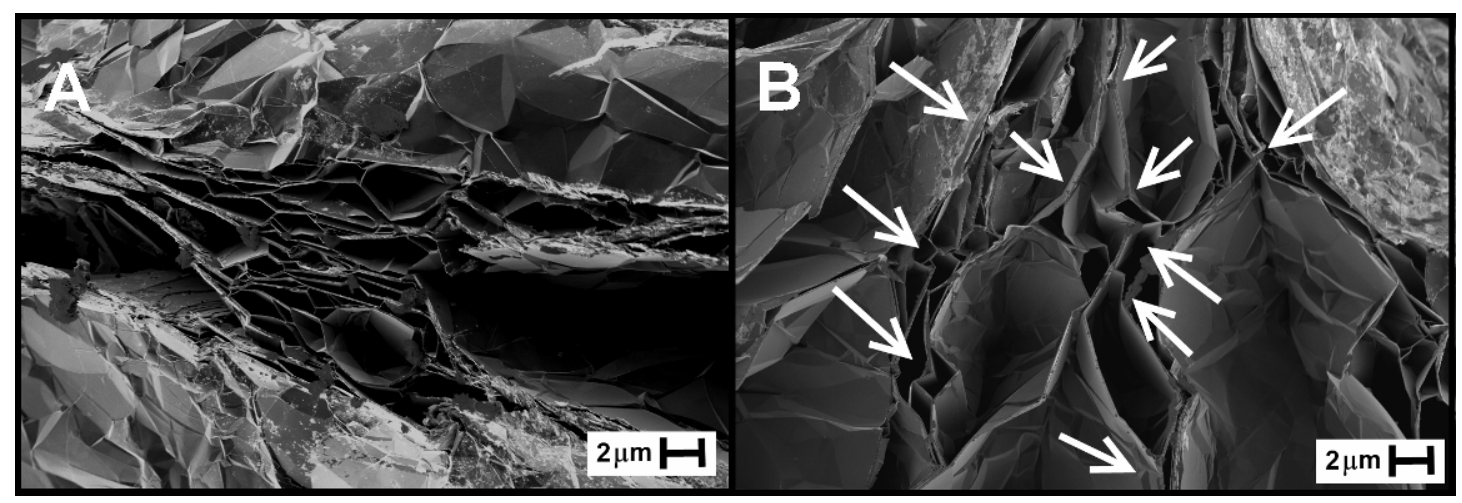

Fig. 18: (A) and (B) Edge structures of commercial exfoliated graphite.

During intercalation, strong oxidizers and acids are used to infiltrate the graphite matrix. These react with the basal plane to produce graphite oxide $[67,68]$. Upon thermal shock, the heterogeneous groups bound to the graphite surface are released in gaseous form. The rapid release of gas drives apart the graphitic layers. It is very likely that the first step during this process is the infiltration of the slit-like pores by the oxidizing medium, followed by reaction with the basal plane. If conditions are inadequate to induce intercalation of the ions into the interlayer spacing, the aforementioned will be the primary source of gas release. When this occurs, the continuous segments will act as bridges which hold different segments together, preventing complete exfoliation and resulting in a highly interlinked structure, as seen in Fig. $18 \mathrm{~A}$ and B.

In effect a single graphite flake is a composite of several smaller polygonal flakes. Each individual flake may be considered a single pristine crystal, with a few discrete screw dislocations and a noteworthy degree of twinning. This is confirmed by observation of large, defect-free and continuous basal surfaces. Graphite is not a typical crystalline solid in the sense that the in-plane interaction is very strong covalent bonding compared with the bonding in metals or the electrostatic bonding in ionic crystals. In plane, graphite has a higher bond strength than diamond. Thus it is 
unlikely that the plane bending caused by the twinning defects will distort the planar hexagonal structure. This is confirmed by the fact that twinning occurs via bond rotation along the armchair crystallographic direction [45]. Hence these defects will not influence inter-atomic distance or lattice plane spacing in the $a-b$ crystallographic directions. These dislocations should also only involve a minor disruption of the lattice in the $c$ direction for a narrow region around the line defect.

Consequently the domain size measurements using both techniques in the $a-b$ crystallographic directions are significantly smaller than expected and this deviation cannot be explained in terms of the crystalline defects. The twinning does however cause a relative shift of the individual flake segments within a larger flake, which is observable by the presence of edge steps and surface ridges. The postulate that segments above and below an edge step have slightly different orientation has recently been confirmed for etch pits in HOPG by the use of dark field tunnelling electron microscopy and electron diffraction [69].

Thus the continuous stacking along the $c$ crystallographic direction is disrupted. Despite being crystalline across the entire height of a flake, the measured pXRD domain size in this direction is significantly lower than the flake thickness and is in rough agreement with the observed step height. Although extensive turbostratic disorder is not evident in this material, the presence of twist grain boundaries cannot be excluded. These stacking faults will result in a similar, segmented disruption of the continuous stacking along the $c$ crystallographic axis and edge step formation.

This illustrative model is only relevant for the material under consideration and will be different for more complex flake geometries [66] or materials which have undergone extensive deformation. In addition, natural graphite crystals from different 
geological settings may have different microstructures from the material considered here due to the diverse growth conditions that are possible.

\subsection{Limitations of the domain size estimates}

The error in estimation of the crystallite size in the $a-b$ crystallographic directions may be attributable to three factors: instrumental limitations, measurement uncertainty and correlation inaccuracy. The limited applicability of pXRD to samples with large crystallites is well known [35]. At a certain value the peak width is governed by the coherence of the incident beam and not by the particle size for perfect crystals [4]. However, the exact numerical value of this limit is difficult to fix. Nonetheless given the error of up to two orders of magnitude, it is highly likely that in this study the estimate is subject to instrumental limitations.

In addition, the pXRD technique was significantly influenced by preferred orientation of the particles. Theoretically, the diffraction line broadening should not be affected by the preferred orientation of the flakes. This is considered a source of error only in intensity measurements for compositional analysis [35]. In extreme cases, however, the reduction may be so severe that distortion of the peak structure could take place. Since the doublet structure is easily discernable for the (110) reflection and the peak position is in good agreement with the theoretically expected value, this is evidently not the case.

Despite the apparent instrumental limitation the FWHM of the silicon standard is still less than the value determined for the natural graphite. Thus the instrumental broadening, measured by the "ideal" silicon standard, is less than the broadening due to the sample and hence the measurement may still be of value. However, measurement uncertainty is of significant concern given the hyperbolic nature of the 
correction for instrumental line broadening. This can be demonstrated by the expression used in the standard method [6] to determine the true FWHM $(\beta)$ from the measured FWHM $(B)$ :

$$
\beta / B=0.9981266-0.0681532 v-2.592769 v^{2}+2.621163 v^{3}-0.9584715 v^{4}
$$

Here $v$ is defined as $b / B$, where $b$ is the measured FWHM for the silicon standard. If required, the measured values must be corrected for doublet separation. For the (110) reflection, the domain size may be calculated as:

$$
L_{A}=11.3 / \beta
$$

At this point Eqn (1) can be substituted into Eqn (2), and the domain size variation plotted as the ratio of the silicon standard FWHM to the carbon, $v$, is varied from zero to one, as demonstrated in Fig. 19, with a suitable selection of the measured FWHM of silicon as $b=0.1$.

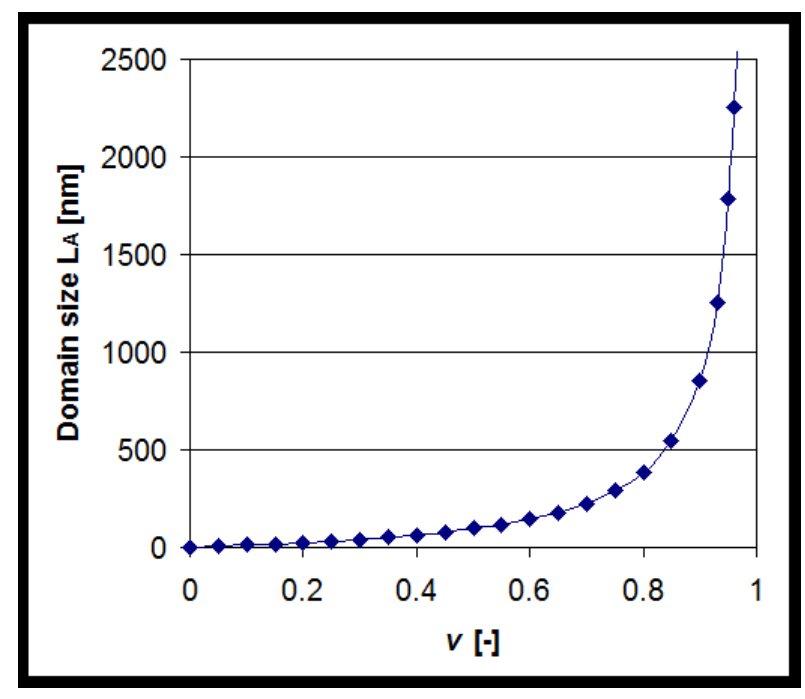

Fig. 19: Relationship between domain size and FWHM ratio for standard method.

As the FWHM of the carbon approaches that of the silicon, a very small change in the ratio results in a massive change for the estimated domain size. Beyond a certain maximum ratio, the variability of the measurement will inhibit the reliable estimation 
of the domain size. The pXRD measurements were repeated five times on exactly the same sample of PRFL. The FWHM ratio of silicon to carbon $(v)$ had a standard deviation of $1.25 \%$. At a ratio of $v=0.8$ this would place the $95 \%$ confidence bounds on the estimated domains size at roughly $\pm 19 \%$ of the estimated value.

This result is completely independent of the equation used to calculate the domain size, in this case Scherrer, and the choice for the value for the FWHM of silicon. These are merely scaling factors for the y-axes values, the percentage change remains the same. The result will only change if the hyperbolic nature of the correction is changed. The relationship given by Eqn (1) was originally derived by Alexander in 1954 [70]. It is based on several improvements to the original postulate by Scherrer in 1918 [71], given by Eqn (3) below:

$$
\beta=B-b
$$

The modification was made to account for seven instrumental weight functions which influence line broadening. However, the differences in these two expressions are small, especially for domain size estimates of large crystallites, as indicated in Fig. $20(\mathrm{~A})$ and $(\mathrm{B})$.

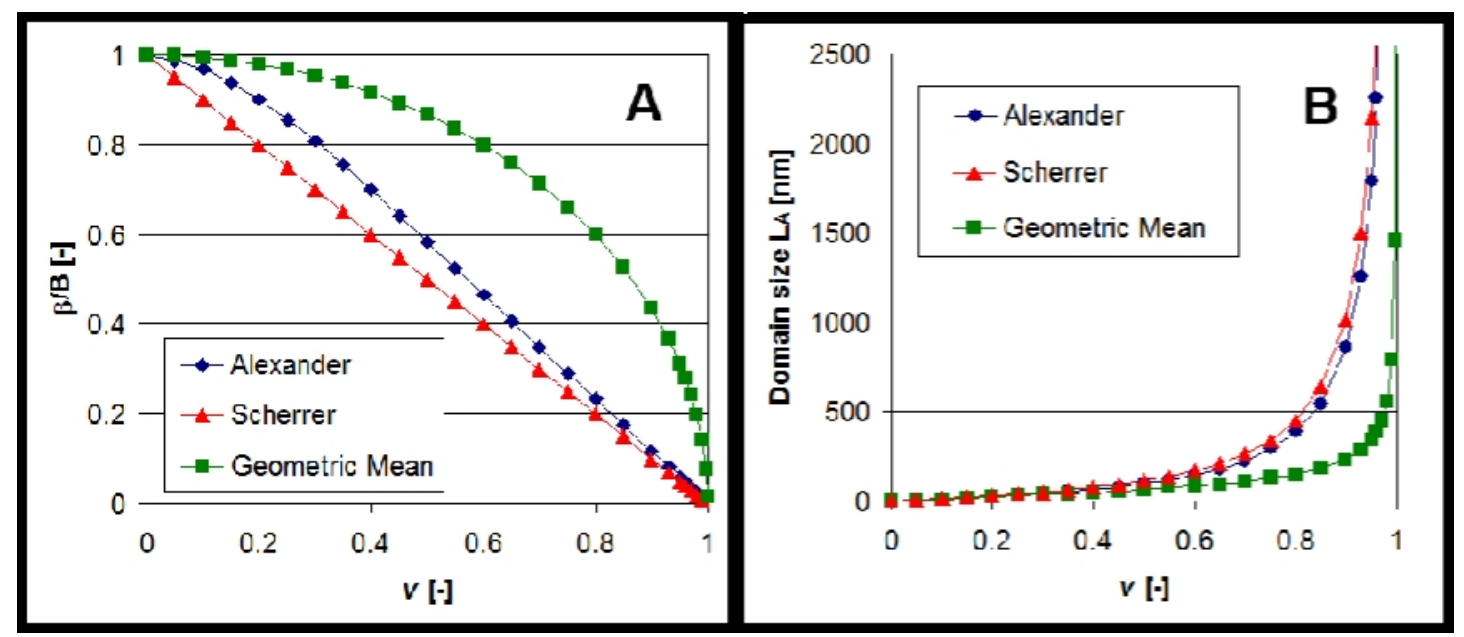

Fig. 20: (A) Correction curves and (B) domain size estimates for Eqn (1), (3) and (4). 
Thus these expressions give similar results at a ratio of $v=0.8$. Unless a new fundamental basis for correcting the measured FWHM for instrumental broadening is proposed, purely from the perspective of measurement uncertainty, this technique should be limited to a maximum ratio of $v=0.8$. In the region of concern for macrocrystalline materials, the error bounds would be on the order of several $100 \%$ of the estimated value. In addition, the relative influence of different defect types or different crystalline arrangements has not been sufficiently investigated, increasing the uncertainty of this estimate.

In some recent studies $[7,57]$ the use of a geometric mean expression is used as correction for the instrumental line broadening, as given by:

$$
\beta=\left(B^{2}-b^{2}\right)^{0.5}
$$

This approach originated from an empirical line profile fitting procedure developed for non-graphitic, or disordered lamellar carbons [72-74]. As such it significantly underestimates domain sizes at high FWHM ratios as shown in Fig. 20 (B) and its use is not recommended for macrocrystalline graphite.

Fixing the maximum allowable FWHM ratio does not necessarily fix the maximum domain size estimate since the correlation accuracy is debatable. From a practical point of view it is convenient to consider the best and worst case scenario implications of the FWHM ratio limit. The shape factor used in the Scherrer equation and hence the standard method, is arbitrarily set to one. This variable can vary by a factor of up to two from 0.9 to 1.84 [35], depending on a variety of considerations.

The instrumental broadening FWHM $(b)$ used to generate the plot given in Fig. 19, was the minimum experimentally obtained value found in the standard method of Iwashita et al. [6]. In the best case scenario, i.e. highest value, for the maximum 
allowable domain size estimate the shape factor will be at a maximum and the instrumental FWHM at a minimum. For these scenarios the instrumental FWHM was varied up and down by a factor of two, to represent a wide range of possible experimental arrangements. The upper and lower bounds on the domain size estimate are shown in Fig. 21.

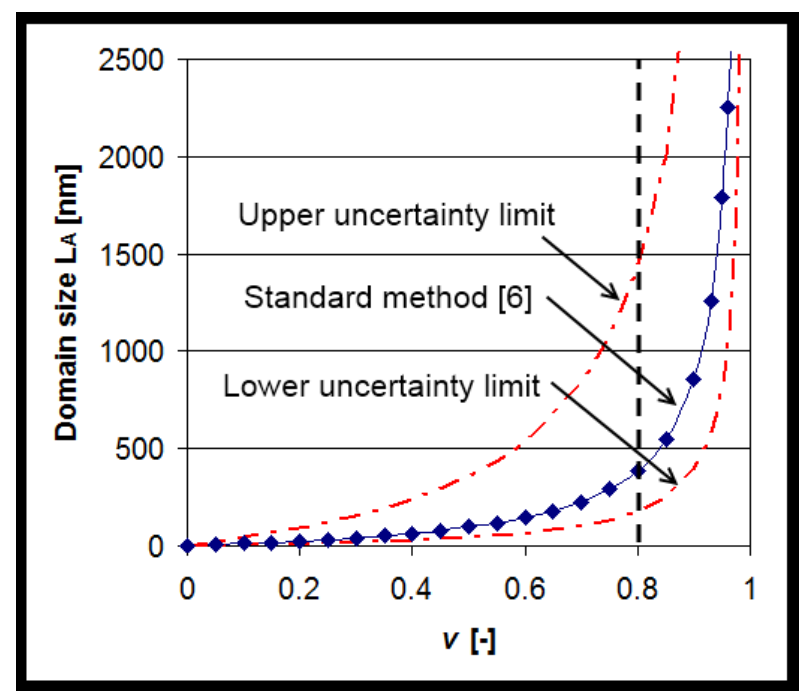

Fig. 21: Upper and lower uncertainty bounds on domain size estimate.

Thus in the best case scenario, the upper limit on the domain size estimate would be $1420 \mathrm{~nm}$ and in the worst case the lower limit is $175 \mathrm{~nm}$. The lower value is close to the maximum recommended limit of $300 \mathrm{~nm}$ suggested by Alexander [70]. The only remaining factor which could influence the line broadening is strain. Due to the heat treatment step the only residual strain in the material was induced by the light mixing in an agate mortar. Given the low in-plane shear strength of graphite, it is more likely that flakes undergo shear slip since this mixing does not have an impact component. The absence of rhombohedral peaks post mixing supports this assertion. Thus it is unlikely that strain is a significant component of line broadening.

The Raman correlation developed by Tuinstra and Koening [38] and adjusted by Cançado et al. [7] to account for different laser energies, is based on the assumption 
that the dominant defects present in the material were crystallite edges. Based on the oxidized microstructure this is clearly not the case for the material under consideration, it is predominantly large crystallites with a few discrete screw dislocations and significant twinning.

For such a material the maximum detectable inter-defect distance will be limited by the spot size of the laser beam, as mentioned by Tuinstra and Koening [38] in 1970. Two types of natural graphite flakes larger than the beam area were shown to have no discernable D peak. The beam used by Tuinstra and Koening had a spot size of $20 \mu \mathrm{m}$, whilst the instrument used in this study has a spot size of around $2 \mu \mathrm{m}$. Thus for the natural graphite flakes under investigation the domain size estimate in the $a-b$ direction is limited by the laser spot size of the instrument.

Given the hyperbolic nature of the Tuinstra and Koening correlation, the upper limit based on measurement uncertainty considerations will be significantly less than the beam size. However, the natural graphite flakes represent a fundamentally different microstructure to the materials studied by Tuinstra and Koenig. Recent work by Lucchese et al. [75] has more in common with the material under consideration. This work involved ion bombardment to induce defects in HOPG and graphene. The average distance between these defects, $L_{D}$, was empirically correlated to the $I(D) / I(G)$ ratio. The fit obtained may be simplified for illustrative purposes by assuming that a single imperfection is only active on one length scale, as opposed to the two used for ion bombardment. By setting the radius of activation, $r_{A}$, equal to radius of structural disordering, $r_{S}$, their expression simplifies to:

$$
I(D) / I(G)=C_{S}\left[1-\exp \left(-\pi r_{S}^{2} / L_{D}^{2}\right)\right]
$$


This model may be used to represent the evolution of the $I(D) / I(G)$ ratio as an increasing number of small point defects are introduced. To represent point defects, the defect radius was chosen to be very small, $r_{S}=0.1 \mathrm{~nm}$. The parameter $\left(C_{S}\right)$, which is defined as the value of the $I(D) / I(G)$ ratio in the highly disordered limit, was adjusted until qualitative agreement with the Tuinstra and Koenig relationship was obtained at $C_{S}=100000$. These two correlations are shown in Fig. 22.

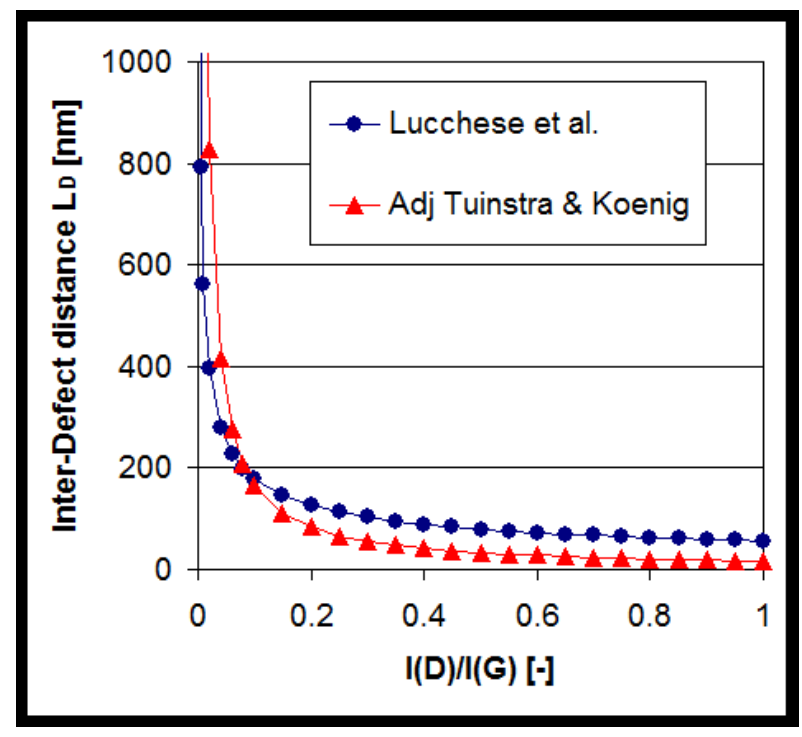

Fig. 22: Comparison of adjusted Tuinstra and Koenig correlation to relationship for discrete defects.

Despite the different defect types used as basis for their formulation, the obtained hyperbolic and exponential behaviours are geometrically quite similar. Thus both would yield comparable confidence bounds on the estimated domain size or interdefect distance. The standard deviation of the D peak measurement in Fig. 5 is roughly $150 \%$ and the domain size estimate was only around $1000 \mathrm{~nm}$. This demonstrates that it would be virtually impossible to accurately obtain domain sizes larger than this using the current correlation, since it would require a peak even smaller than the one measured, assuming the G peak remains the same.

If the height of the $\mathrm{G}$ peak was fixed and a ratio of $I(D) / I(G)=0.1$ used to calculate the D peak, the measured standard deviation would still be $25 \%$ of this 
value. Given the comparatively small value of the ratio, this translates to very large variability in the ratio. However, the $95 \%$ confidence bounds on the estimated domain size at this ratio are roughly $\pm 19 \%$ of the actual value, if the standard deviation of the ratio is limited to $6 \%$. This may be considered as a best case scenario on an improved instrumental setup. These values are for the Tuinstra and Koenig relationship, if Eqn. (5) is used instead, a more conservative value of $\pm 9 \%$ is achieved.

Due to the non-linearity of the correlation, the addition of stress annealed pyrolytic graphite to the linear plot obtained by Tuinstra and Koening is fairly arbitrary. Since the $I(D) / I(G)$ ratio will always tend to zero as $1 / L_{A}$ tends to zero, any highly crystalline graphite with a measurable amount of small defects would give a point that is indistinguishable from the one used. Hence the lowest material of significance used in their study had an $I(D) / I(G)$ ratio of 0.1 , so it is understandable that beyond this value uncertainty considerations become significant.

Intuitively it would be logical that the upper limit for the Tuinstra and Koening correlation should be $20 \mu \mathrm{m}$, however due to the hyperbolic form of the equation this is not the case. This attaches significant ambiguity to the use of this relationship to determine the maximum inter-defect distance or crystallite size corresponding to the $I(D) / I(G)$ ratio limit of 0.1 . Nonetheless using this relationship an upper limit of 165 $\mathrm{nm}$ is found. Furthermore, unlike the pXRD relationship, the fundamental basis for this relationship and hence its geometric shape, has not yet been resolved.

From a physics perspective the D peak is caused by phonon dispersion due to confinement and symmetry breaking as well as defect scattering of double resonance effects $[5,33]$. The exact interpretation of these effects for different defect types is still undecided. For materials with discrete defects the scattering is more closely 
related to the defect density than the inter-defect distance [76]. Work on HOPG has shown a five fold increase in D band intensity when measured edge on as opposed to on the basal surface [77]. This demonstrates that crystallite edges are also Raman active and the laser position on a given sample is critical [78]. For materials with crystallite sizes comparable to the laser beam spot size, grain boundaries in the form of line defects are most relevant. The influence of twinning is unexplored, but given its prevalence and the very small D peak obtained, this work seems to indicate that it is relatively minor.

Based on these considerations it is clear that it is possible to have two graphite materials with identical $I(D) / I(G)$ ratios but entirely different microstructures or defects. Or conversely different $I(D) / I(G)$ ratios can be obtained for the same material depending on the laser position. Understanding the influence and interrelationships of all the different defect structures on the Raman spectra of bulk graphite poses a significant challenge. Technological advances and knowledge gained from nanomaterials may allow the influence of different defects to be identified but unless multiple peak effects can be used to isolate each defect, the complexity, and in some cases anisotropy, of polycrystalline graphite may preclude the use of this technique.

\subsection{Relationship between the domain size estimates and active surface area}

In the context of the proposed model, the practical value of an idealized, true, average crystallite width and height measurement should be considered. In effect a single graphite flake is a composite of several smaller polygonal single crystal flakes. In this case the true crystallite width and height measurements could be regarded as representative of the volume-averaged width and height of these smaller flakes. However, the distribution and interrelated arrangement of these crystals make it 
difficult, if not impossible, to derive a relationship between the average crystallite size of an entire sample and a single, representative particle geometry. Thus it would be challenging to find the direct relationship between this ideal measurement and a material property such as for example the ASA. Given the more complex and dissimilar microstructures found in nuclear grade graphites such as IG-110, PGA, NBG-18, Gilsocarbon, etc. even if the exact measurement of the average domain sizes could be achieved, they should be used with caution as the basis for the comparison of the material properties. In addition, given the considerable uncertainty associated with the current correlations, the ASA estimations using these measurements are subject to significant errors.

For the natural graphite flakes, all the exposed edges will react at the same rate, however, provided significant closed porosity does not exist. Thus from an oxidation or ASA perspective a particle will behave like a distribution of polygons reacting simultaneously, which will lead to an averaging effect. This averaging will be further enhanced by the wide distribution of particles present in a given sample. The polygons are fairly regular and a single particle will always consist of interrelated segments smaller than the overall flake size. Thus it is not unreasonable to deduce that the average polygon size will scale with the average particle size, although perhaps not linearly. As long as the sample chosen is large enough, the effective crystallite width will be related to the average particle diameter. Since the elemental flakes are predominantly stacked on top of each other, the exposed edge area will always be related to the average particle thickness. From a practical perspective, the particle diameter and thickness are be more relevant for ASA estimation, but only once the underlying crystallinity and microstructure of the flakes have been confirmed. 


\section{Conclusions and recommendations}

The microstructure of macrocrystalline graphite is not fully understood. This has, in part, contributed to the uncertainty regarding the meaning of the crystallinity parameters determined by pXRD and Raman spectroscopy. The limitations of these techniques and the suitability of the various correlations utilized to determine these parameters is yet to be unambiguously established. If this dilemma is to be overcome a systematic approach must be adopted. To this end, two widely used procedures for these two techniques were chosen as a starting point. In addition, a cheap and comparatively ideal starting material was selected. Natural flake graphite was secured from a commercial source and purified using a high temperature heat treatment. Residual structural imperfections and beneficiation damage was largely removed by oxidation to retain only the core flake structure.

The objective of the study was to assess the meaning of the calculated crystallographic parameters and shortcomings of the chosen approaches, in light of the microstructure of the idealized, primary flake particles. Using high-resolution SEM imaging the key characteristics of the microstructure were identified and correlated to the crystalline perfection or known defects. Based on this investigation an illustrative model was formulated. An individual flake was found to consist of interrelated stacks of fairly regular polygons each comprising many graphitic layers. These segments may be considered as interwoven single crystals, with a few discrete screw dislocations and a notable degree of twinning. This structure represents an archetypal flake and is not comprehensive for natural graphite which will depend on the material origin and processing.

The twinning disrupts the graphite stacking in the $c$ direction resulting in edge steps. The crystallite domain height measurement is qualitatively representative of the 
average height of a continuously stacked segment. The crystallite domain width measurements are significantly smaller than the observed, intact, undamaged basal surfaces and cannot be accounted for by structural considerations such as defects. This shortcoming is attributed to limitations of the utilized techniques.

The pXRD technique is evidently subject to instrumental limitations due to the large crystallite size. Alternative methods of sample preparation should be explored to circumvent the need for milling whilst decreasing the preferred orientation of the flakes. The hyperbolic shapes of the fundamentally derived correlations lead to significant uncertainty for the domain size estimates. Based on $95 \%$ confidence limits on the estimate of roughly $19 \%$, the correlations should not be used above a FWHM ratio of carbon to silicon of 0.8 . In addition, ambiguity still remains regarding the shape factors for different materials. As such it is recommended that this approach should not be used for materials with domain sizes larger than $1420 \mathrm{~nm}$ and used with caution down to $175 \mathrm{~nm}$.

The Raman technique is directly limited by the spot size of the laser beam for crystallites larger than this value. The microstructure of the natural flakes differs significantly from the materials used to derive the commonly used correlation. Nonetheless recent work indicates that a similar correlation behaviour can be expected. The approach suffers from the same non-linear limitations as pXRD and as such it is not recommended that the technique be used below an $I(D) / I(G)$ ratio of 0.1 . Since the fundamental basis for the utilized correlation has not yet been established, it cannot be reliably used to place a limit on the maximum inter-defect distance or domain size. Nonetheless using this relationship an upper limit of $165 \mathrm{~nm}$ is found. The relationship between the fundamental physics of Raman scattering and different defect types remains unclear. This leads to ambiguous results and unless multiple 
effects can be used to discern between these defects, the technique may have limited applicability for polycrystalline materials which have complex microstructures. It is evident that the Raman technique would benefit from the proposal of a standardized methodology since concerns exists for reproducibility due to flake positioning and orientation, sample temperature due to heating and instrument parameters such as laser polarization and the use of intensity filters.

It is concluded that individual particles can be considered as a single interrelated crystal but due to its composite nature it will be difficult to relate even an ideal, accurate domain size measurement for this material to its ASA. From a practical perspective, the particle diameter and thickness would be more relevant for ASA estimation, but only once the underlying crystallinity and microstructure of the flakes have been confirmed. Thus it seems that future work should rather focus on developing other techniques to estimate ASA, rather than developing models using domain size estimates.

\section{Acknowledgements}

This work is based on research supported by the Skye Foundation and the South African Research Chairs Initiative (SARChI) of the Department of Science and Technology (DST) and the National Research Foundation (NRF). Any opinion, findings and conclusions or recommendations expressed in this material are those of the authors and therefore Skye, the NRF and the DST do not accept any liability with regard thereto.

\section{References}

[1] Reynolds WN. Physical Properties of Graphite. Amsterdam: Elsevier; 1968. 
[2] Radovich LR. Physicochemical properties of carbon materials: a brief overview. In: Serp P, Figueiredo JL, editors. Carbon materials for catalysis, Hoboken, NJ: Wiley; 2009, p. 1-34.

[3] Harris PJF. New perspectives on the structure of graphitic carbons. Crit Rev Solid State Mater Sci 2005;30:235-53.

[4] Dinnebier RE, Billinge SJL. Powder diffraction: theory and practice. Cambridge: Royal Society of Chemistry; 2008.

[5] Ferrari AC. Raman spectroscopy of graphene and graphite: Disorder, electronphonon coupling, doping and nonadiabatic effects. Solid State Commun 2007;143(1-2):47-57.

[6] Iwashita N, Park CR, Fujimoto H, Shiraishi M, Inagaki M. Specification for a standard procedure of X-ray diffraction measurements on carbon materials. Carbon 2004;42(4):701-14.

[7] Cançado LG, Takai K, Enoki T, Endo M, Kim YA, Mizusaki H, et al. General equation for the determination of the crystallite size $\mathrm{L}_{\mathrm{A}}$ of nanographite by Raman spectroscopy. Appl Phys Lett 2006;88(16):163106.

[8] Wissler M. Graphite and carbon powders for electrochemical applications. J Power Sources 2006;156:142-50.

[9] Basini V, Cellier F, Bruguier F, Vitali MP. Selection criteria for chosing carbonaceous matrix for HTR block type design. 3rd International Topical Meeting on High Temperature Reactor Technology. 2006:1-5.

[10] Takeuchi KJ, Marschilok AC, Davis SM, Leising RA, Takeuchi ES. Heattreatment of synthetic graphite under Argon and effect on Li-Ion electrochemistry. Journal of Electrochemical Society. 2005;152:A147-A51. 
[11] Spahr ME, Wilhelm H, Joho F, Panitz J-C, Wambach J, Novak P, et al. Purely hexagonal graphite and the influence of surface modifications on its electrochemical lithium insertion properties. J Electrochem Soc 2002;149:A960-6.

[12] Kercher AK, Nagle DC. M icrostructural evolution during charcoal carbonization by X-ray diffraction analysis. Carbon. 2003;41:15-27.

[13] González D, Altin O, Eser S, Garcia AB. Temperature-programmed oxidation studies of carbon materials prepared from anthracites by high temperature treatment. Materials Chemistry and Physics. 2007;101(1):137-41.

[14] Alonso-Morales N, Gilarranz MA, Heras F, Rodriguez JJ, Eser S. Effects of heat treatment on the structure of LDPE-derived solid carbons. Chem Eng J 2011;172(2-3):1126-36.

[15] Emmerich FG. Evolution with heat treatment of crystallinity in carbons. Carbon. 1995;33:1709-15.

[16] Lu L, Sahajwalla V, Kong C, Harris D. Quantitative X-ray diffraction analysis and its application to various coals. Carbon. 2001;39:1821-33.

[17] Shi H, Barker J, Saidi MY, Koksbang R, Morris L. Graphite structure and lithium intercalation. J Power Sources 1997;68:291-5.

[18] Zaghib K, Nadeau G, Kinoshita K. Effect of graphite particle size on irreversible capacity loss. J Electrochem Soc 2000;147:2110-5.

[19] Zaghib K, Song X, Kinoshita K. Thermal analysis of the oxidation of natural graphite: isothermal kinetic studies. Thermochim Acta 2001;371:57-64.

[20] Contescu CI, Guldan T, Wang P, Burchell TD. The effect of microstructure on air oxidation resistance of nuclear graphite. Carbon. 2012;50(9):3354-66.

[21] Liu Z, Guo Q, Shi J, Zhai G, Liu L. Graphite blocks with high thermal conductivity derived from natural graphite flake. Carbon 2008;46(3):414-21. 
[22] Ong TS, Yang H. Effect of atmosphere on the mechanical milling of natural graphite. Carbon 2000;38:2077-85.

[23] Jiang W, Nadeau G, Zaghib K, Kinoshita K. Thermal analysis of the oxidation of natural graphite - the effect of particle size. Thermochim Acta 2000;351:85-93.

[24] Chung G-C, Jun S-H, Lee K-Y, Kim M-H. Effect of surface structure on the irreversible capacity of various graphitic carbon electrodes. J Electrochem Soc 1999;146:1664-71.

[25] Iwashita N, Inagaki M. Relations between structural parameters obtained by Xray powder diffraction of various carbon materials. Carbon 1993;31:1107-13.

[26] Knight DS, White WB. Characterization of diamond films by Raman spectroscopy. J Mater Res 1989;4:385-93.

[27] Łoś S, Duclaux L, Alvarez L, Hawełek Ł, Duber S, Kempiński W. Cleavage and size reduction of graphite crystal using ultrasound radiation. Carbon. 2013;55:5361.

[28] Wilhelm H, Lelaurain M, McRae E. Raman spectroscopic studies on welldefined carbonaceous materials of strong two-dimensional character. Journal of applied Physics. 1998;84(12):6552-8.

[29] Parthasarathy G, Sreedhar B, Chetty TRK. Spectroscopic and X-ray diffraction studies on fluid deposited rhombohedral graphite from the eastern Ghats Mobile Belt, India. Current Science. 2006;90:995-1000.

[30] Baiju KR, Satish-Kumar M, Kagi H, Nambiar CG, Ravisankar M. Mineralogical characterization of graphite deposits from Thodupuzha-Kanjirappaly belt, Madurai granulite block, southern India. Gondwana Res 2005;8:223-30.

[31] Cazaux J. From the physics of secondary electron emission to image contrasts in scanning electron microscopy. J Electron Microsc (Tokyo) 2012;61(5):261-84. 
[32] Lui J. The versatile FEG-SEM: From ultra-high resolution to ultra-high surface sensitivity. Micros \& Microanal 2008;9:144-5.

[33] Malard LM, Pimenta MA, Dresselhaus G, Dresselhaus MS. Raman spectroscopy in graphene. Phys Rep 2009;473(5-6):51-87.

[34] Ranish JM, Walker PL. Models for roughening of graphite during its catalyzed gasification. Carbon 1990;28:887-96.

[35] Klug HP, Alexander LE. X-ray diffraction procedures for polycrystalline and amorphous materials. New York: Wiley; 1954.

[36] Welham NJ, Williams JS. Extended milling of graphite and activated carbon. Carbon 1998;36:1309-15.

[37] Welham NJ, Berbenni V, Chapman PG. Effect of extended ball milling on graphite. J Alloys \& Compounds 2003;349:255-63.

[38] Tuinstra F, Koenig JL. Raman spectrum of graphite. J Chem Phys 1970;53:1126-30.

[39] Hughes G, Thomas JM, Marsh H, Reed R. Origin of etch pits on graphite surfaces. Carbon 1964;1:339-43.

[40] Palache C. Contributions to the mineralogy of Sterling Hill, New Jersey: morphology of graphite, aresenopyrite, pyrite and arsenic. Am Mineral 1941;26:709-17.

[41] Roscoe C. Catalytic oxidation of Ticonderoga graphite crystals. Carbon $1968 ; 6: 365-72$.

[42] Roscoe C, Thomas JM. The revelations of small-angle boundaries and forest dislocations in graphite monocrystals. Carbon 1966;4:383-90.

[43] Thomas JM. Topographical studies of oxidized graphite surfaces: a summary of the present position. Carbon 1969;7:350-64. 
[44] Rakovan J, Jaszczak JA. Multiple length scale growth spirals on metamorphic graphite $\{001\}$ surfaces studied by atomic force microscopy. American Mineralogist. 2002;87:17-24.

[45] Thomas JM. Microscopic studies of graphite oxidation. In: Walker PL editor. Chemistry and Physics of Carbon, vol 1, New York: Marcel Dekker; 1965, 121203.

[46] Fujita FE, Izui K. Observation of lattice defects in graphite by electron microscopy, Part 1. J Phys Soc Japan 1961;16(2):214-7.

[47] Suarez-Martinez I, Savini G, Haffenden G, Campanera JM, Heggie MI. Dislocations of Burger's Vector $\mathrm{c} / 2$ in graphite. Phys Status Solidi C 2007;4(8):2958-62.

[48] Amelinckz S, Delavignette P, Heerschap M. Dislocations and stacking faults in graphite. In: Walker PL, editor. Chemistry and Physics of Carbon, vol 1; New York: Marcel Dekker, 1965, 1-69.

[49] Nakamizo M, Honda H, Inagaki M. Raman spectra of ground natural graphite. Carbon 1978;16:281-3.

[50] Cong C, Yu T, Sato K, Shang J, Saito R, Dresselhaus GF, et al. Raman characterization of $\mathrm{ABA}-$ and $\mathrm{ABC}$-stacked trilayer graphene. ACS Nano 2011;5(11):8760-8.

[51] Lui CH, Li Z, Chen Z, Klimov PV, Brus LE, Heinz TF. Imaging stacking order in few-layer graphene. Nano Lett 2011;11(1):164-9.

[52] Saito R, Hofmann M, Dresselhaus G, Jorio A, Dresselhaus MS. Raman spectroscopy of graphene and carbon nanotubes. Adv Phys 2011;60(3):413-550. 
[53] Guerin K, Fevrier-Bouvier A, Flandrois S, Couzi M, Simon B, Biensan P. Effect of graphite crystal structure on lithium electrochemical intercalation. J Electrochem Soc 1999;146(10):3660-5.

[54] Parthasarathy G, Sreedhar B, Chetty TRK. Spectroscopic and X-ray diffraction studies on fluid deposited rhombohedral graphite from the eastern Ghats Mobile Belt, India. Curr Sci 2006;90:995-1000.

[55] Hishiyama Y, Nakamura M. X-ray diffraction in oriented carbon films with turbostratic structure. Carbon 1995;33(10):1399-1403.

[56] Li ZQ, Lu CJ, Xia ZP, Zhou Y, Luo Z. X-ray diffraction patterns of graphite and turbostratic carbon. Carbon 2007;45(8):1686-95.

[57] Cançado LG, Takai K, Enoki T, Endo M, Kim YA, Mizusaki H, et al. Measuring the degree of stacking order in graphite by Raman spectroscopy. Carbon $2008 ; 46(2): 272-5$.

[58] Ferrari AC, Meyer JC, Scardaci V, Casiraghi C, Lazzeri M, Mauri F, et al. Raman spectrum of graphene and graphene layers. Phys Rev Lett 2006;97(18): 187401.

[59] Lespade P, Marchand A, Couzi M, Cruege F. Caracterisation de materiaux carbones par microspectrometrie Raman. Carbon 1984;22:375-85.

[60] Pimenta MA, Dresselhaus G, Dresselhaus MS, Cancado LG, Jorio A, Saito R. Studying disorder in graphite-based systems by Raman spectroscopy. Phys Chem, Chem Phys. 2007;9(11):1276-91.

[61] Hennig GR. Electron microscopy of reactivity changes near defects in graphite. In: Walker PL, editor. Chemistry and Physics of Carbon, vol 2, New York: Marcel Dekker; 1966, 1-50. 
[62] Hennig G. Vacancies and dislocation loops in quenched crystals of graphite. J Appl Phys 1965;36(4):1482-6.

[63] Delavignette P, Amelinckx S. Dislocation patterns in graphite. J Nuclear Mater $1962 ; 5: 17-66$.

[64] Luque FJ, Pasteris JD, Wopenka B, Rodas M, Barranechea JF. Natural fluiddeposited graphite: mineralogical characteristics and mechanisms of formation. American Journal of Science. 1998;298:471-98

[65] Friese EJ, Kelly A. Twinning in graphite. Proceedings of the Royal Society of London Series A, Mathematical and Physical Sciences. 1961;264(1317):269-76.

[66] Kvasnitsa VN, Yatsenko VG, Jaszczak JA. Disclinations in unusual graphite crystals from anorthosites of Ukraine. Canadian Mineralogist. 1999;37:951-60. [67] Dideykin A, Aleksenskiy AE, Kirilenko D, Brunkov P, Goncharov V, Baidakova M, et al. Monolayer graphene from graphite oxide. Diamond Relat Mater 2011;20(2):105-8.

[68] Park S, Ruoff RS. Chemical methods for the production of graphenes. Nat Nanotechnol 2009;4(4):217-24.

[69] Delahouzé A, Vignoles GL, Rebillat F, Leyssale J-M, Weisbecker P, et al. Etch pits in graphite oxidized by $\mathrm{O} 2$ : kinetics, morphology, and zigzag/armchair site dynamics. Annual World Conference on Carbon. 2013:37-8.

[70] Alexander L. The synthesis of X-Ray spectrometer line profiles with application to crystallite size measurements. Journal of Applied Physics. 1954;25(2):155-61.

[71] Scherrer P. Bestimmung der Größe und der inneren Struktur von Kolloidteilchen mittels Röntgenstrahlen. Göttinger Nachrichten. 1918;2:98. 
[72] Seehra MS, Pavlovic AS. X-Ray diffraction, thermal expansion, electrical conductivity and optical microscopy studies of coal-based graphites. Carbon. 1993;31(4):557-64.

[73] Perret R, Ruland W. Profile analysis of random-layer lines. Journal of Applied Crystallography. 1968;1:257-62.

[74] Ruland W. Fourier transform methods for random-layer line profiles. Acta Crystallographia. 1967;22:615-23.

[75] Lucchese MM, Stavale F, Ferreira EHM, Vilani C, Moutinho MVO, Capaz RB, et al. Quantifying ion-induced defects and Raman relaxation length in graphene. Carbon 2010;48(5):1592-7.

[76] Araujo PT, Terrones M, Dresselhaus MS. Defects and impurities in graphenelike materials. Mater Today 2012;15(3):98-109.

[77] Kawashima Y, Katagari G. Fundamentals, overtones, and combinations in the Raman spectrum of graphite. Physical Review B. 1995;52:10053-9.

[78] Tan P, Dimovski S, Gogotsi Y. Raman scattering of non-planar graphite: arched edges, polyhedral crystals, whiskers and cones. Philosophical Transactions of the Royal Society A. 2004;362:2289-310. 